\title{
Broadband Passive Targeted Energy Pumping from a Linear Dispersive Rod to a Lightweight Essentially Nonlinear End Attachment
}

\author{
Fotios Georgiades and Alexander F. Vakakis ${ }^{\dagger}$ \\ Mechanics Division, \\ Faculty of Applied Mathematical and Physical Sciences, \\ National Technical University of Athens, \\ fgeorgia@mail.ntua.gr, vakakis@ central.ntua.gr \\ and \\ Gaetan Kerschen \\ Département d'Aérospatiale, Mécanique et Matériaux (ASMA), \\ Université de Liège, \\ g.kerschen@ulg.ac.be; \\ $\uparrow$ Corresponding author, \\ P.O.Box 64042, GR-157 10 Zografos, Athens, Greece \\ Also, \\ Department of Mechanical and Industrial Engineering (adjunct), \\ Department of Aerospace Engineering (adjunct), \\ University of Illinois at Urbana-Champaign, avakakis@uiuc.edu
}

\begin{abstract}
We examine nonlinear resonant interactions between a damped and forced dispersive linear finite rod and a lightweight essentially nonlinear end attachment. We show that these interactions may lead to passive, broadband and on-way targeted energy flow from the rod to the attachment, which acts, in essence, as nonlinear energy sink (NES). The transient dynamics of this system subject to shock excitation is examined numerically using a Finite Element (FE) formulation. Parametric studies are performed to examine the regions in parameter space where optimal (maximal) efficiency of targeted energy pumping from the rod to the NES occurs. Signal processing of the transient time series is then performed, employing energy transfer and/or exchange measures, wavelet transforms, empirical mode decomposition and Hilbert transforms. By computing Intrinsic Mode Functions (IMFs) of the transient responses of the NES and the edge of the rod, and examining resonance captures that occur between them, we are able to identify the nonlinear resonance mechanisms that govern the (strong or weak) one-way energy transfers from the rod to the NES. The present study demonstrates the efficacy of using local lightweight nonlinear attachments (NESs) as passive broadband energy absorbers of unwanted disturbances in continuous elastic structures, and investigates the dynamical mechanisms that govern the resonance interactions influencing this passive nonlinear energy absorption.
\end{abstract}

Keywords: Nonlinear targeted energy pumping, nonlinear resonance, flexible systems 


\section{Introduction}

We study the transient forced dynamics of a linear dispersive rod with a lightweight, essentially nonlinear, ungrounded end attachment. Recent works reported interesting energy exchange phenomena in this type of coupled oscillators, including numerous coexisting branches of fundamental, subharmonic and superharmonic periodic solutions (Lee et al., 2005), and oneway, irreversible transfer of energy from the linear oscillator to the nonlinear attachment, termed targeted nonlinear energy pumping. Such energy exchanges are often associated with transient or sustained resonance captures (Lee et al., 2005; Kerschen et al., 2005; McFarland et al., 2005), whereby the essentially nonlinear attachment engages in transient resonance with the linear oscillator, before the dynamics 'escape' to a different regime of the motion (Arnold, 1988; Quinn, 1997). In cases where the nonlinear attachment acts as passive recipient of vibration energy from the linear oscillator, it essentially acts as nonlinear energy sink (NES).

This work aims to systematically study passive broadband targeted energy transfer from a linear elastic continuum to an attached ungrounded NES. What clearly distinguishes this from previous studies is that due to the essentially nonlinear coupling between the continuum and the NES there is simultaneous nonlinear coupling between the NES and the infinity of modes of the rod, leading to very complicated dynamical interactions. It will be shown, however, that it is precisely due to these complicated dynamic interactions that strong targeted energy transfer phenomena may occur in this system configuration. We mention at this point that although the NES is simultaneously coupled to all modes of the rod, it can only extract energy from one mode at a time; this is due to the fact that the NES can engage in transient resonance capture with only one rod mode at a time, though resonance capture cascades (resulting in sequential energy transfers from sets of rod modes) do occur in this system.

In a related preliminary numerical study, Georgiadis et al. (2005) considered a beam with an attached NES, and demonstrated that targeted energy pumping in that system was indeed possible and, under appropriate design, very efficient. Moreover, in (Vakakis et al., 2004a) the different regimes of dynamic interactions of a semi-infinite dispersive rod with a grounded essentially nonlinear attachment were analytically and numerically studied, but no attempt to study targeted energy pumping was undertaken. In that work it was shown that the attachment

initially engages in nonlinear resonance with incoming traveling elastic waves; as the energy of 
the attachment decreases due to damping and radiation back to the rod, the attachment engages in $1: 1$ resonance capture with the in-phase mode of the dispersive rod, which is the condition under

which targeted energy pumping from the rod to the attachment might occur; further decrease of energy of the attachment leads to escape of the dynamics from resonance capture and settlement of the dynamics to linearized regimes. No study of the efficiency of energy pumping, however, was undertaken in that work. The computational and analytical results in (Vakakis et al., 2004a) reveal that resonant interactions of elastic continua with local essentially nonlinear attachments can give rise to quite complex resonant phenomena, which can be justified by the observation that the local essentially nonlinear attachments considered may introduce global changes in the dynamics of the combined system.

\section{Formulation of the Problem and Methods for Post processing the Numerical Results}

The system under consideration consists of an linear elastic rod of mass distribution $\mathrm{M}$ and length $\mathrm{L}$ resting on an elastic foundation with distributed stiffness $\mathrm{k}$ and distributed viscous damping $\delta$, and coupled to an ungrounded, lightweight end attachment of mass $\mathrm{m}<<\mathrm{M}$ by means of an essentially nonlinear cubic stiffness of constant $\mathrm{C}$, in parallel to a viscous damper $\varepsilon \lambda$ (cf. Figure 1). Assuming that the left boundary of the rod is clamped, that a transient external force $F(t)$ is applied at position $x=d$ of the rod (where $x$ is measured from the left clamped end of the rod), and that the system is initially at rest, the governing equations of motion are given by:

$$
\begin{gathered}
\mathrm{EA} \frac{\partial^{2} \mathrm{u}(\mathrm{x}, \mathrm{t})}{\partial \mathrm{x}^{2}}-\mathrm{ku}(\mathrm{x}, \mathrm{t})-\delta \frac{\partial \mathrm{u}(\mathrm{x}, \mathrm{t})}{\partial \mathrm{t}}+ \\
\mathrm{F}(\mathrm{t}) \delta(\mathrm{x}-\mathrm{d})-\mathrm{C}[\mathrm{u}(\mathrm{L}, \mathrm{t})-\mathrm{v}(\mathrm{t})]^{3} \delta(\mathrm{x}-\mathrm{L})-\varepsilon \lambda\left[\frac{\partial \mathrm{u}(\mathrm{L}, \mathrm{t})}{\partial \mathrm{t}}-\dot{\mathrm{v}}(\mathrm{t})\right] \delta(\mathrm{x}-\mathrm{L})=\mathrm{M} \frac{\partial^{2} \mathrm{u}(\mathrm{x}, \mathrm{t})}{\partial \mathrm{t}^{2}}, 0 \leq \mathrm{x} \leq \mathrm{L} \\
\mathrm{u}(0, \mathrm{t})=0 \\
\mathrm{C}[\mathrm{u}(\mathrm{L}, \mathrm{t})-\mathrm{v}(\mathrm{t})]^{3}+\varepsilon \lambda\left[\frac{\partial \mathrm{u}(\mathrm{L}, \mathrm{t})}{\partial \mathrm{t}}-\dot{\mathrm{v}}(\mathrm{t})\right]=\mathrm{m} \ddot{\mathrm{v}}(\mathrm{t}) \\
\mathrm{u}(\mathrm{x}, 0)=0, \frac{\partial \mathrm{u}(\mathrm{x}, 0)}{\partial \mathrm{t}}=0, \mathrm{v}(0)=0, \dot{\mathrm{v}}(0)=0
\end{gathered}
$$

Moreover, it is assumed that all geometric and material properties of the rod are uniform.

(Figure 1) 
The examination of the equations of motion reveals some challenging aspects of this problem, which are summarized below:

- Due to its essential nonlinearity, the attachment (NES) is directly coupled to all modes of the rod; this is expected to lead to complex nonlinear resonant interactions between the NES and different flexible modes of the rod, which need to be systematically categorized and analyzed.

- Since we aim to study broadband passive targeted energy transfer from the rod to the attachment, it is necessary to consider a strongly (essentially) nonlinear and transient dynamical problem involving a coupled set of partial and ordinary differential equations.

- No mode superposition can be used to express the dynamical response of the linear rod, since the essential stiffness nonlinearity of the attachment amounts to a nonlinear, time-varying boundary condition at its right end.

These challenging aspects dictate a computational approach for solving this problem, together with the use of advanced post processing techniques, capable of decomposing the different nonlinear resonance interactions that are expected to occur between the various modes of the rod and the NES.

The equations of motions were numerically solved with Matlab by employing a finite element (FE) formulation and an implicit time integration scheme based on an adapted Newmark algorithm (Geradin and Rixen, 1997). To ensure proper spatial discretization, a total of $501 \mathrm{FE}$ was used; moreover, the sampling frequency was selected to account for 122 modes of the rod oscillation. At each time step of the numerical integration the total energy balance was computed in order to ensure that, (a) the relative energy error between subsequent steps of the computation was less than $0.001 \%$, and (b) that the error on the conservation of the total energy was less than $1 \%$.

The system parameters were assigned the values:

$$
\mathrm{L}=1, \mathrm{EA}=1.0, \mathrm{M}=1.0, \delta=0.05, \mathrm{~m}=0.1, \varepsilon=0.1, \lambda=0.5
$$

The transient (shock) excitation was chosen to be a half sine pulse of amplitude $\mathrm{F}$ and duration $0.1 \mathrm{~T}_{1}$, where $\mathrm{T}_{1}$ is the period of the first mode of the linear rod; it is assumed that the shock is applied at position $d=0.3$ on the rod. In Table 1 we present the leading eigenfrequencies of the uncoupled and undamped rod (with NES detached) with parameters given by (2) (but zero damping), and elastic foundation equal to $\mathrm{k}=1$.

(Table 1) 
In addition to tracking the total energy of the system at each time step of the computation, we computed also the energy transactions (exchanges) between the linear rod and the NES; in the following results, positive energy transactions correspond to energy flow from the (directly excited) rod to the (initially unexcited) NES, whereas negative energy transactions correspond to backscattering of energy from the NES back to the rod. Clearly, efficient passive targeted energy pumping from the rod to the NES is signified by strong positive energy transactions throughout the transient response of the system.

Post processing of the numerically computed time series of the rod and the NES was performed in two different ways. First, the transient data was analyzed by Wavelet Transforms (WT) employing a Matlab-based algorithm developed at Université de Liège by Dr.V.Lenaerts in collaboration with Dr.P.Argoul from the 'Ecole Nationale des Ponts et Chaussees'. Although the algorithm provides the opportunity to use two kinds of mother wavelets, namely Morlet and Cauchy, in the applications presented herein only the Morlet mother wavelet has been used; this is a Gaussian-windowed complex sinusoid of frequency $\omega_{0}$ (in $\left.\mathrm{rad} / \mathrm{sec}\right), \psi_{M}(\mathrm{t})=\mathrm{e}^{-\mathrm{t}^{2} / 2} \mathrm{e}^{\mathrm{j} \omega_{0} \mathrm{t}}$. The frequency $\omega_{0}\left(\right.$ or $f_{0}$ in $\mathrm{Hz}$ ) is the user parameter which enables one to tune the frequency and time resolution of the results. Occasionally the signals were divided in two phases (earlyand late-phase) for the application of the WT, since as the amplitudes get smaller with respect to their initial values the corresponding wavelet traces are too light to be visible. The WT contour plots (WT spectra) shown below depict the amplitude of the WT of the signal as function of frequency (vertical axis) and time (horizontal axis). Heavy shaded areas correspond to regions where the amplitude of the WT is high, whereas lightly shaded ones correspond to low amplitudes of the WT. Such plots enable one to deduce the temporal evolutions of the dominant frequency components of the signals analyzed, as well as, transitions between different modes that participate in the transient nonlinear responses (Lee et al., 2005; Kerschen et al., 2005).

Further analysis of the numerical time series was performed using a combination of Empirical Mode Decomposition (EMD) and Hilbert Transform. EMD is a method to decompose a signal (time series) in terms components called Intrinsic Mode Functions (IMFs) (Huang et al., 1998; Veltcheva and Soares, 2004; Zhang et al., 2005). The IMFs satisfy the following three main conditions:

- For the duration of the entire time series, the number of extrema and of zero crossings of each IMF should either be equal or differ at most by one 
- At any given time instant, the mean value (moving average) of the local envelopes of the IMFs defined by their local maxima and minima should be zero

- The superposition of all IMFs should reconstructs the time series

Hence, EMD analysis extracts oscillating modulations or modes imbedded in the data. It follows that the essence of the EMD method is to empirically identify the intrinsic oscillatory modes in the data (time series), and to categorize them in terms of their characteristic time scales, by considering the successive extreme values of the signal. The IMFs have usually a physical interpretation as far as their characteristic scales are concerned (indeed, as shown below, certain IMFs possess instantaneous frequencies that are nearly identical to resonance frequencies of the rod or the NES); but this need not always be the case. This implies that certain IMFs may represent artificial (non-physical) oscillating modes of the data. Moreover, as explained in the Appendix, by Hilbert - transforming the IMFs one computes temporal evolutions of their instantaneous amplitudes and frequencies, which, in turn, can be used for the construction of the Hilbert spectrum of the signal (Huang et al., 1998). Nevertheless there are cases where at a certain time scale a transient phenomenon is intermittent - for example, turbulence in fluid motion (Huang et al., 1998, 2003). In these cases, the decomposed components could contain two scales embedded in a single IMF component, and an intermittency criterion should be adopted during the decomposition of the signal (Huang et al., 2003). A synopsis of EMD and the Hilbert transform of the extracted IMFs is presented in the Appendix. In this work these computational approaches were implemented in Matlab

In synopsis, by post processing the transient responses of the rod and the attached NES by means of EMD and Hilbert transform, we aim to study in detail the complex nonlinear resonance interactions taking place between the essentially nonlinear attachment and the various modes of the rod. As we will see in section 5, by combining the results of the Hilbert transform with the corresponding wavelet spectra of the transient responses, we can identify the dominant IMFs of the rod and NES transient responses, and analyze the most important resonance interactions between the rod and the NES that are responsible for the nonlinear energy exchanges between these two subsystems. Hence, we will show that by studying the resonance interactions between dominant IMFs of the NES and the rod responses, we can gain insight into the question of efficiency of passive and irreversible absorption by the NES of broadband vibration energy from the rod. 


\section{Passive Targeted Energy Pumping From the Rod to the NES}

We performed four main sets of FE simulations with parameter values given by (2) and half-sine applied external shock as discussed in the previous section. What distinguished the first and second sets of FE simulations were the parameter values for the elastic foundation of the rod, $\mathrm{k}$, the NES stiffness, C, and the magnitude of the applied shock, F. Specifically, the first set of simulations was performed for a dispersive rod with distributed elastic foundation $\mathrm{k}=1.0$, for 22 values of the nonlinear characteristic in the range $C \in[0.001,20]$, and 15 values of the shock amplitude in the range $\mathrm{F} \in[0,500]$. This gave a total of $22 \times 15=330$ possible pairs $(\mathrm{C}, \mathrm{F})$, all of which were simulated in the first series. Similarly, the second set of numerical simulations involved the same 330 numerical simulations but for elastic foundation $\mathrm{k}=0$, corresponding to a nondispersive rod. Each of the transient simulations of the first two series was performed for a sufficiently large time interval, so that at the end of the simulation at least $99 \%$ of the input shock energy was damped by the distributed viscous damping of the rod and the discrete viscous damper of the NES. This damped energy measure ensured that no dynamics was missed in the transient simulations due to insufficient time of integration.

(Figure 2)

In Figure 2 we depict contour plots of the percentage of shock energy eventually dissipated by the damper of the NES as function of the parameters $C$ and F for the first two series of FE simulations. This energy measure is computed by the relation:

$$
\eta \equiv\left(\lim _{\mathrm{t}>>1} \frac{\int_{0}^{\mathrm{t}} \varepsilon \lambda\left[\dot{\mathrm{v}}(\tau)-\frac{\partial \mathrm{u}(\mathrm{L}, \tau)}{\partial \tau}\right]^{2} \mathrm{~d} \tau}{\int_{0}^{\mathrm{T}} \mathrm{F}(\tau) \frac{\partial \mathrm{u}(\mathrm{d}, \tau)}{\partial \tau} \mathrm{d} \tau}\right) \times 100
$$

The integral in the numerator represents the energy dissipated by the damper of the NES up to time instant $\mathrm{t}$; the integral in the denominator (with upper limit $\mathrm{T}$ equal to the duration of the applied shock) represents the total input energy applied to the rod by the external shock. Given that the system under consideration is dissipative, it holds that for $t>>1$ the measure $\eta$ should approach a definite asymptotic limit, which represents the percentage of input energy that is 
eventually absorbed and dissipated by the NES. In the remainder of this work, wherever we mention the energy measure $\eta$, we will be implying its long-term asymptotic limit.

Clearly, regions of the plots of Figure 2 where the energy measure $\eta$ is relatively large correspond to strong and effective targeted energy pumping from the rod to the NES, as a significant percentage of the shock energy of the rod is eventually absorbed and dissipated by the NES. The numerical results of Figure 2 reveal that when strong shocks are applied (high input energy), enhanced energy transfers occur (with more than $75 \%$ of shock energy eventually dissipated by the NES) when the essential stiffness nonlinearity is relatively weak. By contrast, when smaller shocks are applied, strong targeted energy transfers occur $(\eta>75 \%)$ over a wide range of values of the essentially nonlinear stiffness of the NES. This should be expected, since when the energy is high, a stiff essential nonlinearity amounts to a near-rigid connection between the rod and the NES, leading to small relative velocities across the NES damper, and thus, to small energy dissipation capacity of the NES. An additional remark regarding the plots of Figure 2 is that there are only small quantitative and qualitative differences between the dispersive and nondispersive cases, indicating that the efficiency of targeted energy pumping from the rod to the NES is rather insensitive to the existence or absence of dispersion in the elastic continuum.

Two additional sets of FE simulations were performed for both dispersive $(\mathrm{k}=1)$ and nondispersive $(\mathrm{k}=0)$ rods. In each of these sets we considered three distinct values of the nonlinear stiffness of the NES, namely, $\mathrm{C}=0.004,2$ and 10, and varied the mass of the NES in the range $\mathrm{m} \in[0.01,0.1]$ (for a total of 11 values), and the input amplitude in the range $\mathrm{F} \in[1,420]$ (for a total of 13 values). Therefore for each value of $\mathrm{C}$ there were $11 \times 13=143$ possible pairs $(\mathrm{m}, \mathrm{F})$, all of which were realized in the numerical simulations. Again, to ensure that the numerical integration was of sufficient duration, we imposed the requirement that at least $99 \%$ of the shock energy should be dissipated at the end of each of FE simulation.

As in the previous two sets of simulations, we observed only small differences in the energy dissipation plots between the dispersive and nondispersive cases; hence, only the results for the dispersive rod will be discussed from here on. In Figure 3 we depict the NES energy dissipation measure $\eta$ [relation (3)] as function of the NES mass $m$ and the shock strength F, for four chosen values of the nonlinear stiffness characteristic $(C=0.004,0.01,2$ and 10). As in Figure 2, we deduce that there are parameter regions where strong targeted energy pumping from 
the rod to the NES occurs, as indicated by the high values of $\eta$ (over $75 \%$ at certain parameter ranges). Moreover, as the value of the nonlinear stiffness characteristic, $\mathrm{C}$, increases the region of relatively strong targeted energy pumping shifts to smaller shock strengths and becomes narrower. In addition, in parameter ranges where relatively strong targeted energy pumping occurs there appears to be nearly negligible dependence of the NES energy dissipation measure $\eta$ on the NES mass $m$, for values of $m>0.02$.

(Figure 3)

These results indicate that the essentially nonlinear attachment (the NES) can be designed to passively absorb and locally dissipate a significant portion of the applied (broadband) shock energy in the rod. Moreover, the NES can be designed so that the strong energy pumping is robust to small changes in the input energy and the system parameters. These results demonstrate the efficacy of using lightweight essentially nonlinear local attachments as passive absorbers and local energy dissipaters of broadband energy from elastic continua. This result extends analogous results reported in previous works where discrete linear oscillators with local essentially nonlinear attachments were studied. In the next section we proceed to a detailed analysis of the dynamics that govern strong targeted energy pumping in the system of Figure 1.

\section{Nonlinear Resonance Interactions between the Rod and the NES}

Considering the case when the rod is dispersive $(\mathrm{k}=1)$, we considered in detail $21 \mathrm{FE}$ simulations (termed from now on 'Applications') in the first set of results (cf. Figure 2). In Table 2 we present the system parameters used for each Application, together with the NES energy dissipation measure $\eta$ and the types of phenomena observed: ' $B$ ' indicates the occurrence of nonlinear beat phenomena in the transient responses of the rod and the NES; ' $\mathrm{I}$ ' indicates irreversible (one-way) energy transfer from the rod to the NES (however, even in these cases there exists a very small initial region where very early nonlinear beat phenomena occur, but this region is small, so we may designate the phenomenon as being predominantly irreversible energy transfer); whereas, 'B-I' indicates early nonlinear beat phenomena in the transient dynamics, followed by irreversible energy transfer from the rod to the NES. A simple comparison of the different Applications listed in Table 2 reveals that, with the exception of 7, 16, and 21, all Applications correspond to rather strong energy pumping, as a major part of the input (broad- 
band) vibration energy in the rod is passively absorbed by and dissipated at the NES. This observation is itself interesting since it shows that strong energy pumping in the system under consideration occurs for wide combinations of input energy and system parameters. It follows that comparisons between energy pumping efficiency between different Applications can only be performed on a relative basis, and in that context the measure $\eta$ can only be considered as a relative indicator of efficiency of energy pumping. Specific examples for all three types of the aforementioned dynamics ('B', 'B-I' and 'I') are discussed below.

(Table 2)

The Applications listed in Table 2 are partitioned into three main groups. Group (a) consists of Applications 3,4,5,8,17,19 and 20 with relatively strong energy transfers from the rod to the NES and relatively small input energies (shocks). All three dynamical mechanisms (B, I, and B-I) are realized in the Applications of Group (a). The second group of Applications $1,2,9,10,11,12,14$ and 15 (labelled Group (b)) is again characterized by relatively strong energy transfers and higher levels of input energy; these Applications involve the dynamical mechanisms B and B-I. Finally, Group (c) consists of Applications 6,7,13,16,18 and 21 with relatively weak energy transfers and higher levels of input energy; all Applications in this Group are characterized by persistent nonlinear beat phenomena (mechanism B), involving continuous energy exchanges between the rod and the NES.

Typical transient responses of the edge of the rod and of the NES are depicted in Figures 4 and 5, with the corresponding relative displacements of the NES with respect to the edge of the rod presented in Figures 6 and 7. In each of these plots (as in the following ones) each Application is characterized by its Group and the governing dynamical mechanism (for example, in Figure 4, Application 1 is labelled by (b,B), and so on). The measure of relative displacement between the NES and the edge of the rod affects directly the efficiency of targeted energy pumping, since the capacity of the NES to dissipate energy transferred from the rod is directly related to the relative velocity across its viscous damper; it follows that enhanced energy dissipation by the NES is achieved when this relative displacement (and its derivative) attains large magnitudes, especially in the critical initial regime of the motion where the energy is still relatively large (and energy dissipation due to damping in the rod is still small). Examples of large, early relative displacement between the rod and the NES are Applications 1 (Fig.6) and 2 (Fig.7) with corresponding energy dissipation percentages of $\eta=76 \%$ and $72 \%$, respectively, 
whereas an example of small relative displacement is Application 7 (Fig. 7) corresponding to $\eta=51 \%$.

An interesting feature of the NES is its capacity to interact with more than one structural modes of the rod (this is done sequentially through resonance capture cascades, see below). Indeed, due to the essential coupling nonlinearity, the NES is simultaneously 'coupled' to all modes of the rod (as can be realized from the differential equations (1)), and, hence, it has the capacity to nonlinearly resonate with structural modes over wide frequency ranges (provided, of course, that the initial conditions are appropriate). Such multi-modal interactions of the NES with the rod may lead to multi-frequency energy pumping, or to complex dynamic phenomena, such as, abrupt transitions between different dynamical regimes (Vakakis et al., 2004b; Lee et al., 2005). These interactions become apparent in the wavelet transform spectra of the dynamics, although in some cases they may be visible in the time series themselves (for instance, in Figure 6 - Application 1 the frequency content of the NES is rich, and the resonance capture cascade is evident; this is also the case in Figure 7 - Application 14).

(Figures 4,5,6,7)

A useful computational tool for studying the dynamic interaction between the rod and the NES is to consider the transient energy transaction history between these two subsystems. In Figure 8 we depict the energy transaction histories between the NES and the rod for Applications 1 and 17, where strong targeted energy pumping from the rod to the NES occurs $(76 \%$ of shock energy dissipated by the NES in Application 1, and 71\% in Application 17). Note in these plots the strong positive spikes of energy transmission from the rod to the NES, and the small negative spikes of energy backscattered from the NES to the rod, which explains the relatively high values of the energy dissipation measure $\eta$ realized in these Applications. In addition, in both Applications there is a positive net balance of energy transferred from the rod to the NES during the critical early regime of the response where the overall energy of the motion is relatively high since energy dissipation by the dampers of the rod is relatively small. In Figure 9 we depict the energy transaction histories for two Applications (7 and 21) where relatively weak targeted energy pumping occurs (51\% of shock energy eventually dissipated by the NES in Application 7, and $21 \%$ in Application 21); note in these simulations the strong backscattering of energy from the NES to the rod, which explains the weaker energy transfers in this case. Moreover, in all simulations considered, the energy exchanges between the rod and the NES are realized in the 
form of spikes, which reflects the fact that the external excitation itself is in the form of a spike (short pulse), that generates forward - and backward - propagating pulses in the rod which are, either reflected at the left (clamped) boundary of the rod, or are partially reflected and transmitted into the NES at its right boundary.

It should be clear that an alternating series of positive and negative spikes of energy transfers is an indication that nonlinear beat phenomena between the rod and the NES occur (dynamical mechanism B in Table 2). This is especially evident in the energy transaction history of Application 1 (cf. Figure 8), where nonlinear beat phenomena with strong positive spikes are clearly detected. In Application 17 (cf. Figure 8) the series of strong initial nonlinear beats is followed by irreversible (one-way) energy transfer (dynamical mechanism I in Table 2) from the rod to the NES, as evidenced by the late series of positive - only energy spikes.

Similar persisting nonlinear beats are observed in the energy transaction histories depicted in Figure 9 where Applications with relatively weaker targeted energy pumping are depicted. The distinctive feature of the beats in these cases is that the negative and positive energy spikes are of comparable magnitudes, preventing strong 'flow of energy' from the rod to the NES. Finally, in Figure 10 we depict the energy transaction for Application 20 where irreversible energy transfers from the rod to the NES occur right from the beginning of the dynamics, and nonlinear beat phenomena are completely absent; indeed, in Applications 8 and 20 there is only irreversible 'flow of energy' from rod to the NES, where the energy is localized at the NES and finally dissipated by the NES damper. The resulting targeted energy pumping is relatively strong in this case, and comparable to the strong energy pumping results for Applications governed by the dynamical mechanisms B and B-I.

(Figures 8,9,10)

In Figure 11 we depict the Wavelet Transform (WT) spectra of the relative transient responses between the edge of the rod (from now referred to as 'the rod') and the NES, for four cases where strong (cases (a,B-I) - Application 17, (a,B) - Application 3, and (a,I) - Application 20), and weaker (case (c,B) - Application 7) targeted energy pumping occurs. The WT spectra reveal the dominant frequency components of the corresponding responses, as well as their temporal evolutions with decreasing energy due to damping dissipation.

Regarding Application 17 (case (a,B-I)) where strong targeted energy pumping occurs (cf. Figure 11a), we observe early (e.g., high energy) transient resonant interactions of the NES 
with predominantly the first and second modes of the rod, as well as a weaker early NES resonant interaction with the third mode of the rod; all these early interactions are in the form of nonlinear beats. Moreover, we observe a nonlinear transition of the dominant frequency component of the dynamics to a nonlinear mode whose frequency shifts below the first linearized mode of the rod. During this low-frequency transition the dynamics localizes gradually to the NES with decreasing energy due to damping dissipation; similar transitions were found in previous works (Lee et al., 2005; Kerschen et al., 2005) where the dynamics of discrete linear oscillators coupled to NESs was analytically and computationally examined. The aforementioned early resonant interactions explain the nonlinear beats observed in the early response (mechanism 'B'), whereas the low frequency transition of the dominant harmonic produces one-way irreversible energy transfers from the rod to the NES (mechanism 'I') in this Application.

(Figure 11)

Similar transition of the dynamics to a nonlinear mode is observed in the WT plot of Figure 11b (Application $3-(a, B)$ ), however, in this case the frequency variation of the nonlinear mode (dominant harmonic) takes place between the first and second eigenfrequencies of the rod. Similarly to Application 17 (Figure 11a), this transition results in strong targeted energy pumping from the rod to the NES. Additional early beats between the NES and the second and third modes of the rod and the NES take place (mechanism 'B', as in Application 17); more importantly, however, there occurs a secondary late transition of the dynamics from the nonlinear mode to the first rod mode, after which additional persistent beats between the NES and the first rod mode take place (mechanism ' $\mathrm{B}$ '). This late transition is qualitatively different from the dynamics depicted in Figure 11a.

No such low frequency transitions occur in the WTs of the relative transient responses of Applications 7 (case (c,B) - weaker targeted energy pumping) and 20 (case (a,I) - strong targeted energy pumping), presented in Figures 11c and d, respectively. In the case of weaker energy pumping (Figure 11c) we observe strong and persistent resonance locking of the relative response at the second linearized mode of the rod, which explains the corresponding persistent nonlinear beats observed in the transient response. It is interesting to note that in this case there is complete absence of resonance interactions between the relative response and the first mode of the rod. In the case of stronger targeted energy pumping in Application 20 (Figure 11d) there is similar resonance locking of the relative response at the first linearized mode of the rod, 
which, however, is not as persistent as in the WT of Figure 11c. In both cases, there is no transition of the early (high energy) relative motion to a nonlinear mode localized at the NES (as in Figures 11a,b).

As a final remark concerning the WT spectra of Figure 11, we note clearly the multimodal content of the dynamics of rod-NES interaction, reaffirming our previous comment on the capacity of the NES to sequentially interact with a set of linearized modes of the rod. In general, such multimodal resonant interactions enhance the effectiveness of nonlinear energy pumping in the system, and lead to complex dynamical phenomena such as resonance capture cascades. A more detailed dynamic analysis of such multi-modal resonance interactions will be presented in a future work. The WT spectra of Figures 11, when combined with Empirical Mode Decomposition (EMD) analysis of the transient responses of the rod and the NES form a powerful computational tool that can be utilized to reveal additional features of the resonance interactions that occur between the rod and the NES. This is discussed in what follows.

Representative results of EMD analysis for Applications 7, 17 and 20 are listed in Table 3. To increase the accuracy of the analysis, the early and late transient responses of Application 7 were analyzed separately, whereas, no such separation was deemed necessary for the other two Applications. In each case we analyzed through EMD the transient responses of the edge of the rod and of the NES. Examination of the IMFs of these transient responses and their instantaneous frequencies provides insightful information concerning the resonance interactions between the rod and the NES. Indeed, the computation of the instantaneous frequencies of the IMFs, combined with the WT spectra depicted in Figure 11 provide us with the opportunity to interpret the WT results in terms of resonance interactions between specific IMFs of the rod and the NES. In what follows we will apply this methodology to examine in detail resonance interactions in Applications 17 (case (a,B-I)) and 7 (case (c,B)).

In Figure 12a we present IMF - based reconstructions of the transient responses of the edge of the rod and the NES for Application 17; complete agreement between numerical simulation and IMF - based reconstruction is observed, proving the validity of the EMD analysis for decomposing the transient nonlinear responses through IMFs. Representative IMFs are depicted in Figure 12b. Next, decompositions of the IMFs in terms of their instantaneous amplitudes and phases were performed in order to examine their individual frequency contents. This information should be analyzed together with the corresponding WT spectrum of the 
relative transient response between the edge of the rod and the NES (cf. Figure 11a); in that plot it is clearly observed that in this case strong nonlinear energy pumping is associated with a low frequency 'locking' of the dynamics to a nonlinear mode below the first eigenfrequency of the $\operatorname{rod}(0.29 \mathrm{~Hz})$. In Figure 13a we depict the transient evolutions of the IMF frequency components of the rod and the NES, e.g., the instantaneous frequencies $\dot{\phi}_{2 \mathrm{NES}}(\mathrm{t})$ and $\dot{\phi}_{9 \mathrm{Rod}}(\mathrm{t})$, respectively, superimposed to the wavelet spectra of the respective numerical time series. Several conclusions can be drawn from these results:

(i) It is clear that the $2^{\text {nd }}$ IMF of the NES and the $9^{\text {th }}$ IMF of the rod possess nearly constant instantaneous frequencies precisely at the low frequency range of the nonlinear mode of the WT spectrum of Figure 11a; hence, these IMFs engage in 1:1 resonance capture in the initial (high energy) stage of the transient dynamics.

(ii) This 1:1 resonance capture becomes apparent by considering the corresponding phase plot of the phase difference $\phi_{2 \mathrm{NES}}(\mathrm{t})-\phi_{9 \mathrm{Rod}}(\mathrm{t})$ in the early time window where the $1: 1$ resonance capture occurs (cf. Figure 13b). Indeed, resonance capture between two IMFs is indicated by the non time - like, 'slow' evolution of the difference between their corresponding phase difference, so that the averaging theorem cannot be applied with respect to that phase difference and preventing averaging out of the dynamics. It is precisely such resonance captures that lead to passive targeted energy pumping from the rod to the NES, as quantified by the energy dissipation measure $\eta$ (Lee et al., 2005; Kerschen et al., 2005). Moreover, the fact that the described 1:1 resonance capture takes place in the early stage of the dynamics where the energy of the system is high, explains the strong targeted energy pumping observed in this Application.

(iii) In the mentioned resonance capture regime, the $2^{\text {nd }}$ (dominant) IMF of the rod coincides in frequency with the dominant harmonic component of the transient response of the NES, whereas the $9^{\text {th }}$ IMF of the rod coincides with the lowest of the dominant harmonic components of the transient response of the edge of the rod.

These results (together the ones presented below) demonstrate the capacity of the EMD-WT analysis to accurately 'pinpoint' the oscillatory components of the rod and NES time series that engage in resonance capture, and, are responsible for the passive energy pumping phenomena from the rod to the NES.

(Figures 12,13) 
In Figure 14a we depict the simulated and IMF - based reconstructed responses for Application 7 (case (c,B) - weaker targeted energy pumping), from which again complete agreement between simulations and IMF - reconstructions is observed. Representative IMFs of the early (high energy) responses of the edge of the rod and the NES are depicted in Figure 14b. Consideration of the resonance interactions between the IMFs of the rod and the NES reveals the reason behind the weak targeted energy pumping in this Application. Referring to the WT spectrum of the relative response between the edge of the rod and the NES for this Application (cf. Figure 11c), we established 'locking' of the dynamics in the vicinity of the second linearized eigenfrequency of the rod (close to $0.77 \mathrm{~Hz}$ ). Examining the temporal evolutions of the instantaneous frequencies of the IMFs of the early transient responses of the edge of the rod and the NES, Figures $15 \mathrm{a}$ and $15 \mathrm{~b}$, respectively, we note that the $1^{\text {st }}$ IMF of the NES and the $5^{\text {th }}$ IMF of the rod develop delayed frequency 'plateaus' close to $0.77 \mathrm{~Hz}$ for $\mathrm{t}>12$ (cf. Figures 15a,b). Moreover, examining the phase plot of the phase difference $\phi_{1 \mathrm{NES}}(\mathrm{t})-\phi_{5 \mathrm{Rod}}(\mathrm{t})$ over the time window where the frequency plateaus are realized, we observe the characteristic loops that indicate a clear 1:1 resonance capture between these two IMFs. However, since this resonance capture occurs at a later stage of the response (e.g., at a stage where a significant portion of the initial energy of the system has already been dissipated due to damping), the resulting targeted energy pumping from the rod to the NES is not as strong as in the previously discussed Application 17, where the corresponding resonance capture takes place at the critical early stage of the motion where the energy of the system is at its highest level. In Figure 15 we also show that in Application 7 there occurs an additional 'delayed' $1: 1$ resonance capture between the $2^{\text {nd }}$ IMF of the NES and the $6^{\text {th }}$ IMF of the edge of the rod at a frequency near the first eigenfrequency of the rod $(0.29 \mathrm{~Hz})$, which, however, does not lead to significant energy transfer from the rod to the NES. Finally, from Figures 15a,b we note that, by superimposing the instantaneous IMF frequencies to the WT spectra of the respective numerical time series, we infer that the $1^{\text {st }}$ and $2^{\text {nd }}$ IMFs of the NES coincide with the higher and lower dominant harmonics, respectively, of the time series of the NES, but only during the later stage of the motion. Similar conclusions can be drawn with regard to the $5^{\text {th }}$ and $6^{\text {th }}$ IMF of the rod.

(Figures 14,15)

Summarizing, it appears that strong targeted energy pumping in the system under consideration is associated with resonance captures between IMFs of the NES and rod responses 
at specific frequency ranges and at the critical early stage of the motion where the energy level is high; delayed resonance captures between IMFs of the rod and the NES that occur at diminished energy levels result in weaker targeted energy pumping from the rod to the NES. In terms of the corresponding WT spectra, strong energy exchanges and early resonance captures between IMFs are associated with 'locking' of the dynamics with nonlinear normal modes that localize at the NES as the energy of the system diminishes due to damping dissipation.

\section{Discussion}

The results of this work demonstrate the efficacy of using lightweight essentially nonlinear attachments - termed Nonlinear Energy Sinks (NESs) - as passive absorbers of broadband (shock) energy from elastic structures. The resulting irreversible targeted energy pumping of shock energy to the NESs, eliminate in an effective way unwanted structural disturbances. Hence, the proposed design provides a new paradigm for passive shock isolation of elastic structures.

An interesting (and appealing) feature of the NES concept is that, although an NES represents a local alteration of the physical configuration of a structure, it can affect the global structural dynamics. The reason behind this seemingly paradoxical finding (and also the basic feature that distinguishes the NES from previous absorber designs of the literature), is the essential stiffness nonlinearity of the NES, which enables its resonance interactions (resonance captures) with structural modes at arbitrary frequency ranges, provided, of course, that the point of attachment is not close to nodes of the structural modes of interest. In addition, the lack of a preferential resonance frequency of the NES enables it to engage in nonlinear resonance with a series of structural modes, passively extracting energy from each mode before shifting in frequency (due to decreasing energy) and engaging in resonance with the next; such resonance capture cascades were computationally studied in previous works (Vakakis et al., 2004b; Kerschen et al., 2005), and result in multi-frequency targeted energy pumping.

An additional new feature presented in this work is the use of combined Wavelet Transforms (WTs) and Empirical Mode Decomposition (EMD) as a tool for identifying the specific resonance captures responsible for the nonlinear interactions (and the passive irreversible energy transfers) between the NES and the structure to which it is attached. The 
analysis presented herein indicates that strong targeted energy pumping from the structure to the NES are associated with 1:1 resonance captures between oscillatory Intrinsic Mode Functions (IMFs) of the two structural components, occurring during the initial, high energy stage of the dynamics. In contrast, weak targeted energy pumping is associated with delayed 1:1 resonance captures between IMFs, occurring at later regimes of the dynamics, where the energy has been significantly reduced due to damping dissipation. Future systematic work needs to be performed to verify this interesting conjecture.

It was found that there exist at least three distinct dynamical mechanisms governing the NES - rod nonlinear resonance interactions; namely, nonlinear beat phenomena (mechanism 'B'), direct one-way irreversible energy transfers from the rod to the NES (mechanism 'I'), and a combination of the two (mechanism 'B-I'). Although no direct association of any one of these three mechanisms to the efficiency of energy pumping of the NES can be discerned based on the results presented in this work, some interesting observations based on the previous computational findings can still be made. Indeed, from the results listed in Table 2 we note that nearly all (with the exception of Applications 8 and 20) relatively high values of the percentage of energy dissipated at the NES, $\eta$, are associated with the occurrence of early nonlinear beats in the response (cases ' $\mathrm{B}$ ' and 'B-I'); this is not to say, however, that nonlinear beats always give rise to relatively high values of $\eta$ (counterexamples are Applications 7, 16, 18 and 21 in the same Table). These observations regarding early nonlinear beats are consistent with earlier results reported in (Kerschen et al., 2005). In that work it was found that the most efficient nonlinear energy pumping in a linear oscillator coupled to an ungrounded NES is always 'triggered' by early nonlinear beats; this occurred, however, only when these nonlinear beats took place in a certain area of the frequency-energy plot of the periodic orbits of the underlying hamiltonian system (in essence, these beats acted as 'bridging orbits' that 'guided' the dynamics of the system to a state where strong energy pumping to the NES occurred). Away from this area of the frequency - energy plot (or away from the corresponding ranges of parameters) early nonlinear beats were counterproductive, producing weak energy interactions between the linear and nonlinear subsystems, and eventually, inefficient energy pumping. Returning to the results reported in this work and motivated by the previous discussion, we conjecture that strong energy pumping in the rod-NES configuration is similarly 'triggered' by early nonlinear beat phenomena occurring in a certain area of the frequency - energy plot of the underlying hamiltonian 
system (e.g., of the undamped rod with undamped attached NES). To prove this conjecture one needs to follow certain steps; first to construct the nonlinear frequency - energy plot of the periodic (and quasi-periodic) orbits of the underlying hamiltonian system (a challenging task in itself); then, compute periodic and quasi-periodic orbits with initial conditions that 'trigger' strong energy pumping; finally, superimpose the computed frequency - energy plot to wavelet transform spectra of the numerical transient responses of the damped system (similarly to the methodology followed in (Kerschen et al., 2005) for discrete systems) that would prove that transient responses producing strong energy pumping are 'triggered' by periodic or quasiperiodic nonlinear beats in a certain area of the frequency - energy plot.

In synopsis, this work presents an extension of the concept of NES to continuous elastic structures, and provides basic computational tools for analyzing the nonlinear dynamics that influence the capacity of the NES to passively absorb and dissipate shock energy from these structures. Ongoing work focuses on the application of the NES concept to eliminate unwanted disturbances (and instabilities) that occur in practical structures modeled by finite elements, and on the development of NESs with alternative configurations that improve their robustness and their capacity to absorb broadband energy from flexible structures. 


\section{References}

Arnold V.I. (ed.), 1988. Dynamical Systems III, Encyclopaedia of Mathematical Sciences, Springer Verlag, Berlin and New York

Georgiades F., A.F. Vakakis, 2005. Dynamics of a Linear Beam with an Attached Local Nonlinear Energy Sink. Communications in Nonlinear Science and Numerical Simulation (Rapid Communication) (in press).

Geradin M., Rixen D., 1997. Mechanical Vibrations, Theory and Application to Structural Dynamics, John Willey \& Sons, New York.

Huang N.E., Z. Shen, S.R. Long, M.C. Wu, H.H. Shih, Q. Zheng, N.C. Yen, C.C. Tung, H.H. Liu, 1998. The Empirical Mode Decomposition and the Hilbert Spectrum for Nonlinear and Nonstationary Time Series Analysis, Proceedings of the Royal Society of London, Series A, Vol. 454 pp. 903-995.

Huang N.E., , M.C. Wu, S.R. Long, S.S.P. Shen, W. Qu, P. Gloersen, K.L. Fan, 2003. A Confidence Limit for the Empirical Mode Decomposition and Hilbert Spectral Analysis, Proceedings of the Royal Society of London, Series A, Vol. 459, pp. 2317-2345.

Kerschen G., Y.S. Lee, A.F. Vakakis, D.M. McFarland, L.A. Bergman, 2005. Irreversible Passive Energy Transfer in Coupled Oscillators with Essential Nonlinearity. SIAM Journal on Applied Mathematics, Vol. 66, No.2, pp. 648-679.

Lee Y.S., G. Kerschen, A.F. Vakakis, P.N. Panagopoulos, L.A. Bergman, D.M. McFarland, 2005. Complicated Dynamics of a Linear Oscillator with a Light, Essentially Nonlinear Attachment, Physica D, Vol. 204, No.1-2, pp. 41-69.

McFarland D.M., G. Kerschen, J. Kowtko, Y.S. Lee, L.A. Bergman, A.F. Vakakis, 2005. Experimental Investigation of Targeted Energy Transfers In Strongly and Nonlinearly Coupled Oscillators, Journal of the Acoustical Society of America, Vol. 118, No. 2, pp. 791-799.

Quinn, D., 1997, Resonance Capture in a Three Degree of Freedom Mechanical System, Nonlinear Dynamics, Vol. 14, pp. 309-333.

Vakakis A.F., L.I. Manevitch, A. Musienko, G. Kerschen, L.A. Bergman, 2004a. Transient Dynamics of a Dispersive Elastic Wave Guide Weakly Coupled to an Essentially Nonlinear End Attachment. Wave Motion, Vol. 41/2, pp. 109-132.

Vakakis A.F., D.M. McFarland, L.A. Bergman, L.I. Manevitch, O. Gendelman, 2004b, Isolated Resonance Captures and Resonance Capture Cascades Leading to Single- or Multi-Mode Passive Energy Pumping in Damped Coupled Oscillators, Journal of Vibration and Acoustics Vol. 126, No.2, pp. 235-244. 
Veltcheva A.D., C.G. Soares, 2004. Identification of the Components of Wave Spectra by the Hilbert Huang Transform Method. Applied Ocean Research, Vol. 26, pp. 1-12.

Zhang R.R., R. King, L. Olson, Y.-L. Xu, 2005. Dynamic Response of the Trinity River Relief Bridge to Controlled Pile Damage: Modeling and Experimental Data Analysis Comparing Fourier and Hilbert-Huang Techniques. Journal of Sound and Vibration, Vol. 285, pp. 10491070. 


\section{Appendix: Empirical Mode Decomposition (EMD) and Hilbert Transforms of the extracted IMFs}

The EMD algorithm for computing the intrinsic mode functions (IMFs) of a signal (time series) $\mathrm{x}(\mathrm{t})$ involves the following steps (Huang et al., 1998):

- Consider separately the envelopes defined by the local maxima and minima of $x(t)$. Interpolate the locus of all local maxima of $\mathrm{x}(\mathrm{t})$ through a spline approximation, thus constructing an upper envelope of the signal; similarly interpolate the locus of all local minima of $x(t)$ thus creating a lower envelope of the signal.

- Compute the moving average $\mathrm{m}_{1}(\mathrm{t})$ between the lower and the upper envelopes, and define the modified, zero-mean signal $h_{1}(t)=x(t)-m_{1}(t)$.

- Repeat this procedure $\mathrm{k}$ times starting from $\mathrm{h}_{1}(\mathrm{t})$ until the signal computed at the $\mathrm{k}$-th iteration, say $\mathrm{h}_{1 \mathrm{k}}(\mathrm{t}) \equiv \mathrm{c}_{1}(\mathrm{t})$, satisfies the properties of an IMF. This holds, provided that the following standard deviation between the (k-1)-th and k-th steps,

$$
\mathrm{SD}=\sum_{\mathrm{t}=0}^{\mathrm{T}}\left[\frac{\left|\mathrm{h}_{1(\mathrm{k}-1)}(\mathrm{t})-\mathrm{h}_{1(\mathrm{k})}(\mathrm{t})\right|^{2}}{\mathrm{~h}_{1(\mathrm{k}-1)}^{2}(\mathrm{t})}\right], \quad \mathrm{T} \text { signal duration }
$$

lies between a preset tolerance, which in this work was chosen in the range $[0.2,0.3]$. Practically, this criterion implies that the k-th iteration $\mathrm{h}_{1 \mathrm{k}}(\mathrm{t}) \equiv \mathrm{c}_{1}(\mathrm{t})$ is approximately (within a tolerance) zero-mean. This process yields the first IMF of the signal $x(t)$, namely $\mathrm{c}_{1}(\mathrm{t})$.

- The second-order remainder of the signal, $\mathrm{x}_{2}(\mathrm{t})$, is defined by the relation $\mathrm{x}_{2}(\mathrm{t})=\mathrm{x}(\mathrm{t})-\mathrm{c}_{1}(\mathrm{t})$, on which the previous procedure is repeated to extract the second $\operatorname{IMF~} \mathrm{c}_{2}(\mathrm{t})$.

- The outlined procedure is repeated until the $n$-th order remainder $x_{n}(t)$ becomes a monotonic function of time.

By construction, the lowest order IMFs contain the oscillatory components of the signal with the highest frequency components; as the orders of the IMFs increase, their frequency contents also decrease. 
After the EMD of the time series, the extracted IMFs are Hilbert transformed in order to compute their approximate transient amplitudes and phases. The Hilbert transform $\mathrm{H}[\mathrm{c}(\mathrm{t})] \equiv \hat{\mathrm{c}}(\mathrm{t})$ of a signal (time series) $\mathrm{c}(\mathrm{t})$ is defined as follows,

$$
\hat{\mathrm{c}}(\mathrm{t})=\left(\frac{1}{\pi}\right) \int_{-\infty}^{+\infty} \frac{\mathrm{c}(\tau)}{\mathrm{t}-\tau} \mathrm{d} \tau \equiv \frac{1}{\pi \mathrm{t}} * \mathrm{c}(\mathrm{t})
$$

where $(*)$ denotes the convolution operator. In the context of the following analysis, the Hilbert transform of the signal $\mathrm{c}(\mathrm{t})$ can be regarded as the 'imaginary' part of the signal, enabling one to complexify the signal. Indeed, defining the complexified analytical signal,

$$
\psi(t)=c(t)+j \hat{c}(t)
$$

we compute its amplitude $\mathrm{A}(\mathrm{t})$ and phase $\varphi(\mathrm{t})$ by expressing the complexification in polar form:

$$
\psi(t)=A(t) e^{j \varphi(t)}=A(t) \cos \varphi(t)+j A(t) \sin \varphi(t)
$$

It follows that the signal can be represented in the form,

$$
\mathrm{c}(\mathrm{t})=\mathrm{A}(\mathrm{t}) \cos \varphi(\mathrm{t})
$$

with amplitude and phase given by:

$$
\mathrm{A}(\mathrm{t})=\sqrt{\mathrm{c}(\mathrm{t})^{2}+\hat{\mathrm{c}}(\mathrm{t})^{2}}, \quad \varphi(\mathrm{t})=\tan ^{-1}\left(\frac{\hat{\mathrm{c}}(\mathrm{t})}{\mathrm{c}(\mathrm{t})}\right)
$$

The representations $(\mathrm{A}-5,6)$ enable one to compute the instantaneous frequency of the signal $\mathrm{c}(\mathrm{t})$ according to the following definition:

$$
\mathrm{f}(\mathrm{t})=\frac{\dot{\varphi}(\mathrm{t})}{2 \pi}=\frac{\mathrm{c}(\mathrm{t}) \dot{\hat{\mathrm{c}}}(\mathrm{t})-\hat{\mathrm{c}}(\mathrm{t}) \dot{\mathrm{c}}(\mathrm{t})}{2 \pi\left[\mathrm{c}(\mathrm{t})^{2}+\hat{\mathrm{c}}(\mathrm{t})^{2}\right]}
$$

It is precisely these results that make the combined EMD - Hilbert Transform useful for the problem considered in this work. Indeed, the decomposition of the rod and NES transient responses in terms of their oscillatory components (the IMFs), and the subsequent computation of their instantaneous frequencies, provide a useful tool for studying nonlinear resonant interactions between the NES and the various modes of the rod. To this end, we say that a $k: m$ resonance capture occurs between an IMF of the $\operatorname{rod} \mathrm{c}_{1}(\mathrm{t})$ and an IMF of the NES $\mathrm{c}_{2}(\mathrm{t})$ with phases $\varphi_{1}(\mathrm{t})$ and $\varphi_{2}(\mathrm{t})$, respectively, whenever their instantaneous frequencies satisfy the following approximate relation: 


$$
\mathrm{kj} \&(\mathrm{t})-\operatorname{mj} \&_{2}(\mathrm{t}) \gg \text { const, for } \mathrm{t} \Xi\left[\mathrm{T}_{1}, \mathrm{~T}_{2}\right]
$$

The time interval $\left[\mathrm{T}_{1}, \mathrm{~T}_{2}\right]$ defines the duration of the resonance capture. As shown in the next section, a more complete picture of the resonance capture between the two mentioned IMFs can be gained by constructing appropriate phase plots that involve the phase difference $\Delta \varphi_{12}(\mathrm{t}) \equiv \varphi_{1}(\mathrm{t})-\varphi_{2}(\mathrm{t})$ and its derivative. More specifically, a resonance capture is signified by the existence of a small loop in the phase plot of $\Delta \varphi_{12}(\mathrm{t})$ versus $\Delta \dot{\varphi}_{12}(\mathrm{t})$, whereas absence of (or escape from) resonance capture is signified by time-like (that is, monotonically varying) behavior of $\Delta \varphi_{12}(\mathrm{t})$ and $\Delta \dot{\varphi}_{12}(\mathrm{t})$. In addition, the ratio of instantaneous frequencies of the IMFs, $\dot{\varphi}_{1}(\mathrm{t}) / \dot{\varphi}_{2}(\mathrm{t})$, provides an estimate of the order of the resonance capture k:m. 


\section{Tables}

Table 1: The leading eigenfrequencies of the uncoupled dispersive undamped $\operatorname{rod}(\mathrm{k}=1)$

\begin{tabular}{|c|c|c|c|c|c|c|c|c|}
\hline Mode & 1 & 2 & 3 & 4 & 5 & 6 & 7 & 8 \\
\hline Eigenfrequencies $(\mathrm{Hz})$ & 0.29 & 0.77 & 1.26 & 1.76 & 2.26 & 2.75 & 3.25 & 3.75 \\
\hline
\end{tabular}

Table 2: FE simulations of the first series studied in detail

\begin{tabular}{|cccccc|}
\hline $\begin{array}{c}\text { FE Simulation - } \\
\text { Application No }\end{array}$ & Phenomena & Group & C & F & $\eta(\%)$ \\
1 & B & b & 0.02 & 180 & 76 \\
2 & B-I & b & 0.004 & 180 & 72 \\
3 & B & a & 6 & 10 & 76 \\
4 & B & a & 9 & 10 & 74 \\
5 & B & a & 2 & 20 & 75 \\
6 & B & C & 5 & 50 & 60 \\
7 & B & C & 5 & 100 & 51 \\
8 & I & a & 0.01 & 20 & 67 \\
9 & B & b & 0.01 & 260 & 76 \\
10 & B & b & 0.005 & 400 & 75 \\
11 & B & b & 0.02 & 200 & 75 \\
12 & B & b & 0.06 & 100 & 76 \\
13 & B & C & 0.08 & 420 & 60 \\
14 & B & b & 0.09 & 100 & 74 \\
15 & B & b & 0.1 & 60 & 74 \\
16 & B & c & 0.2 & 460 & 52 \\
17 & B-I & a & 0.8 & 10 & 71 \\
18 & B & c & 0.8 & 180 & 56 \\
19 & B-I & a & 5 & 3 & 69 \\
20 & I & a & 0.2 & 5 & 67 \\
21 & B & C & 20 & 500 & 21 \\
\hline
\end{tabular}


Table 3: Number of IMFs used in the EMD of each application

\begin{tabular}{|c|c|c|c|c|}
\hline Application & $\begin{array}{c}\text { No of IMFs in } \\
\text { Early Rod } \\
\text { Response }\end{array}$ & $\begin{array}{c}\text { No of IMFs in } \\
\text { Late Rod } \\
\text { Response }\end{array}$ & $\begin{array}{c}\text { No of IMFs in } \\
\text { Early NES } \\
\text { Response }\end{array}$ & $\begin{array}{c}\text { No of IMFs in } \\
\text { Late NES } \\
\text { Response }\end{array}$ \\
\hline $7-(\mathrm{c}, \mathrm{B})$ & $\begin{array}{c}15 \\
(0<\mathrm{t}<45)\end{array}$ & $\begin{array}{c}12 \\
(45<\mathrm{t}<80)\end{array}$ & $\begin{array}{c}12 \\
(0<\mathrm{t}<45)\end{array}$ & $\begin{array}{c}10 \\
(45<\mathrm{t}<80)\end{array}$ \\
\hline $17-(\mathrm{a}, \mathrm{B}-\mathrm{I})$ & $\begin{array}{c}16 \\
(0<\mathrm{t}<30)\end{array}$ & - & $\begin{array}{c}9 \\
(0<\mathrm{t}<30)\end{array}$ & - \\
\hline $20-(\mathrm{a}, \mathrm{I})$ & $\begin{array}{c}15 \\
(0<\mathrm{t}<35)\end{array}$ & - & $\begin{array}{c}10 \\
(0<\mathrm{t}<35)\end{array}$ & - \\
\hline
\end{tabular}




\section{Figure Captions}

1. Linear elastic rod with essentially nonlinear end attachment (NES).

2. Contour plots of the percentage of shock energy eventually dissipated by the NES, $\eta$, as function of the nonlinear stiffness, $\mathrm{C}$, and the strength of the shock, F, for both dispersive $(\mathrm{k}=1)$ and nondispersive $(\mathrm{k}=0)$ rods.

3. Contour plots of the portion of shock energy eventually dissipated by the NES, $\eta$, as function of the NES mass, $\mathrm{m}$, and the strength of the shock, F; three different values of the nonlinear stiffness, $\mathrm{C}$, are considered, and a dispersive rod $(\mathrm{k}=1)$ is assumed.

4. Responses of the end of the rod and of the NES for applications 1,17,20 (first series of FE simulations - Table 2).

5. Responses of the end of the rod and of the NES for applications 2,7,14,19 (first series of FE simulations - Table 2).

6. Relative responses between the end of the rod and the NES for applications 1,17,20 (first series of FE simulations - Table 2).

7. Relative responses between the end of the rod and the NES for applications 2,7,14,19 (first series of FE simulations - Table 2).

8. Energy transaction history between the rod and the NES for applications 1 (case 'B') and 17 (case 'B-I') where strong targeted energy pumping from the rod to the NES occurs; the series of nonlinear beat phenomena are clearly visible.

9. Energy transaction history between the rod and the NES for applications 7 and 21 (cases 'B') where weak targeted energy pumping from the rod to the NES occurs; note the persistent series of nonlinear beat phenomena that prevent irreversible energy transfer to the NES.

10. Energy transaction history between the rod and the NES for application 20 (case 'I') where strong but nonoptimal energy pumping from the rod to the NES occurs; note the absence of nonlinear beat phenomena, and the immediate irreversible energy transfers to the NES right from the beginning of the motion.

11. Morlet Wavelet transform of the relative motion of the NES with respect to the edge of the rod: (a) Application 17 - case 'B-I'; (b) Application 3 - case 'B'; (c) Application 7 - case 'B'; (d) Application 20 - case ' $I$ '. The first three eigenfrequencies of the uncoupled rod are indicated.

12. EMD analysis of Application 17 - case 'B-I': (a) IMF-based reconstructed transient responses of the edge of the rod and the NES versus numerical simulations; (b) Selected IMFs of the transient responses of the edge of the rod and the NES.

13. Nonlinear 1:1 resonance capture in Application 17 between the $2^{\text {nd }}$ IMF of the NES and the $9^{\text {th }}$ IMF of the edge of the rod: (a) Instantaneous frequencies of the two IMFs; (b) phase plot of the phase difference indicating the 1:1 resonance capture.

14. EMD analysis of Application 7 - case 'B': (a) IMF-based reconstructed transient responses of the edge of the rod and the NES versus numerical simulations - early and late responses are treated separately; (b) Selected IMFs of the early transient responses of the edge of the rod and the NES.

15. Nonlinear 1:1 resonance captures in Application 7 between the $1^{\text {st }}$ IMF of the NES and the $5^{\text {th }}$ IMF of the edge of the rod, and the $2^{\text {nd }}$ IMF of the NES and the $6^{\text {th }}$ IMF of the rod: (a) Instantaneous frequencies of the IMFs of the NES; (b) Instantaneous frequencies of the IMFs of the edge of the rod; (c) phase plots of the phase differences indicating the two 1:1 resonance captures. 
Figures

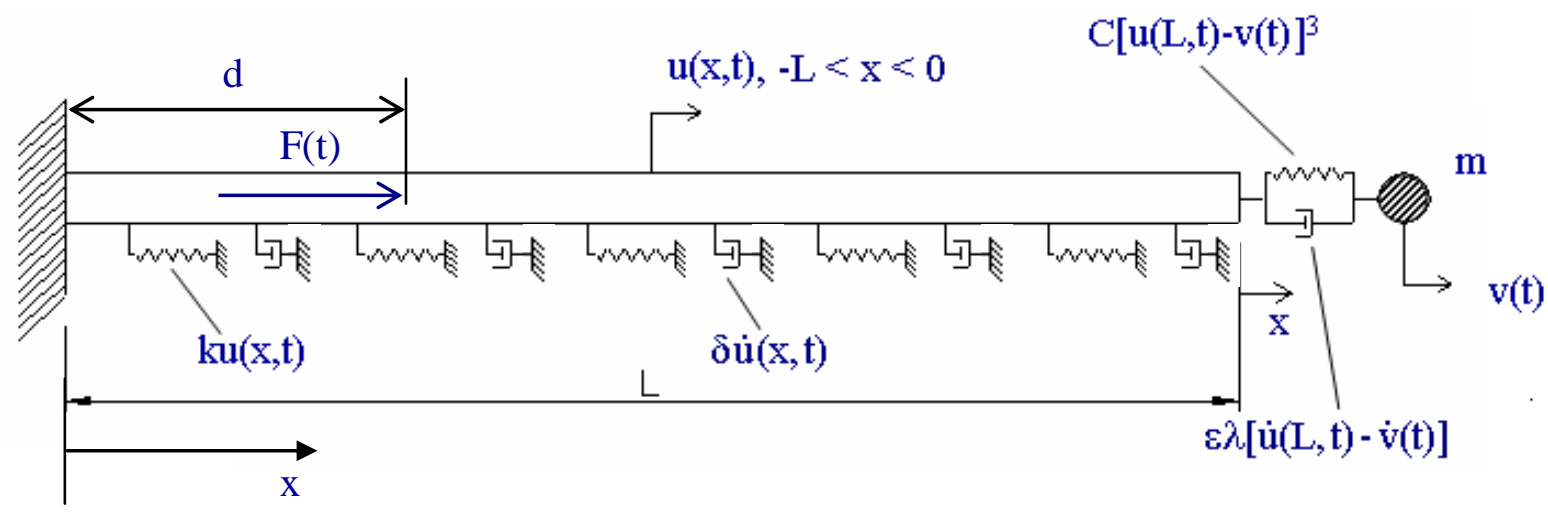

Figure 1 

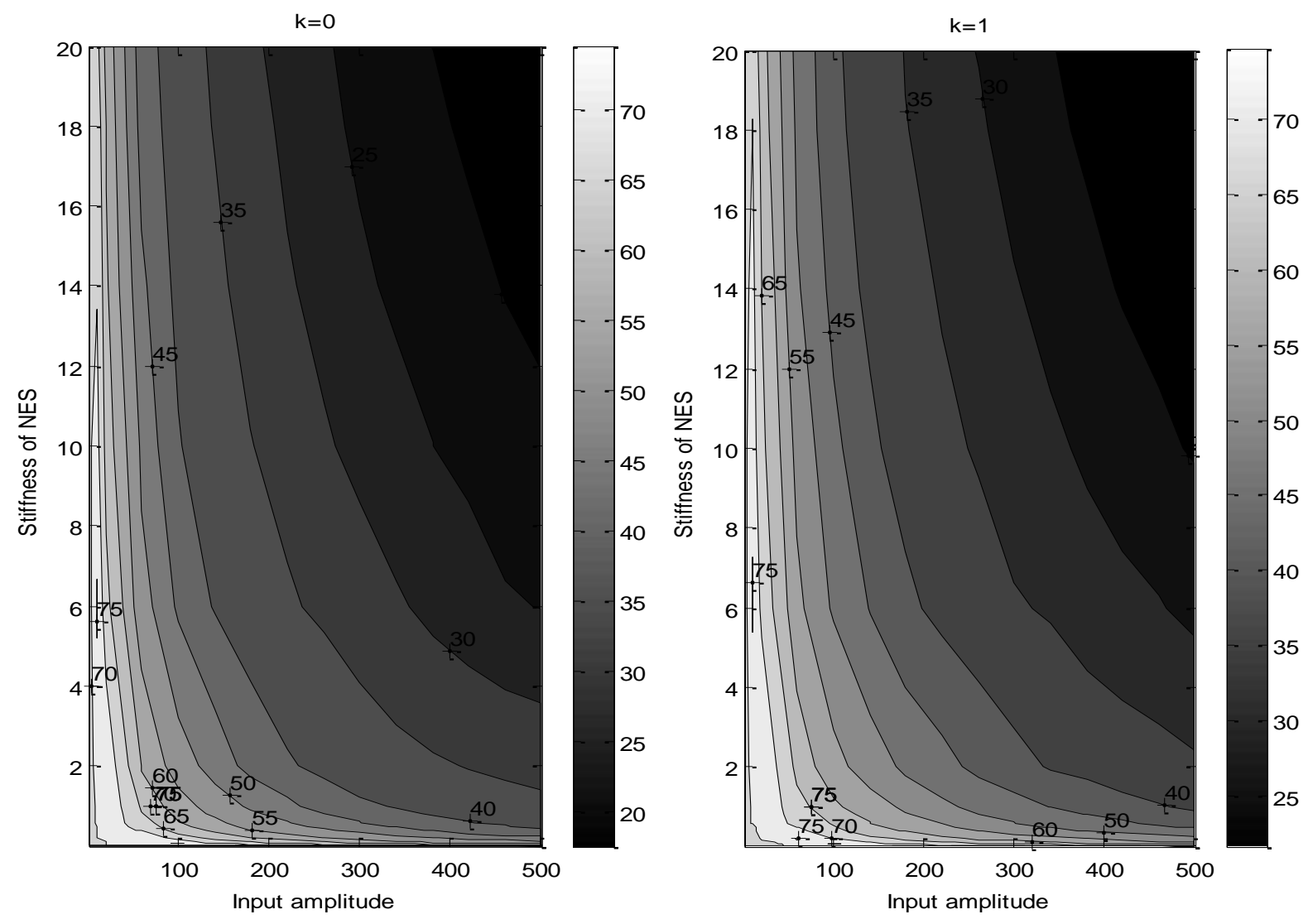

Figure 2 

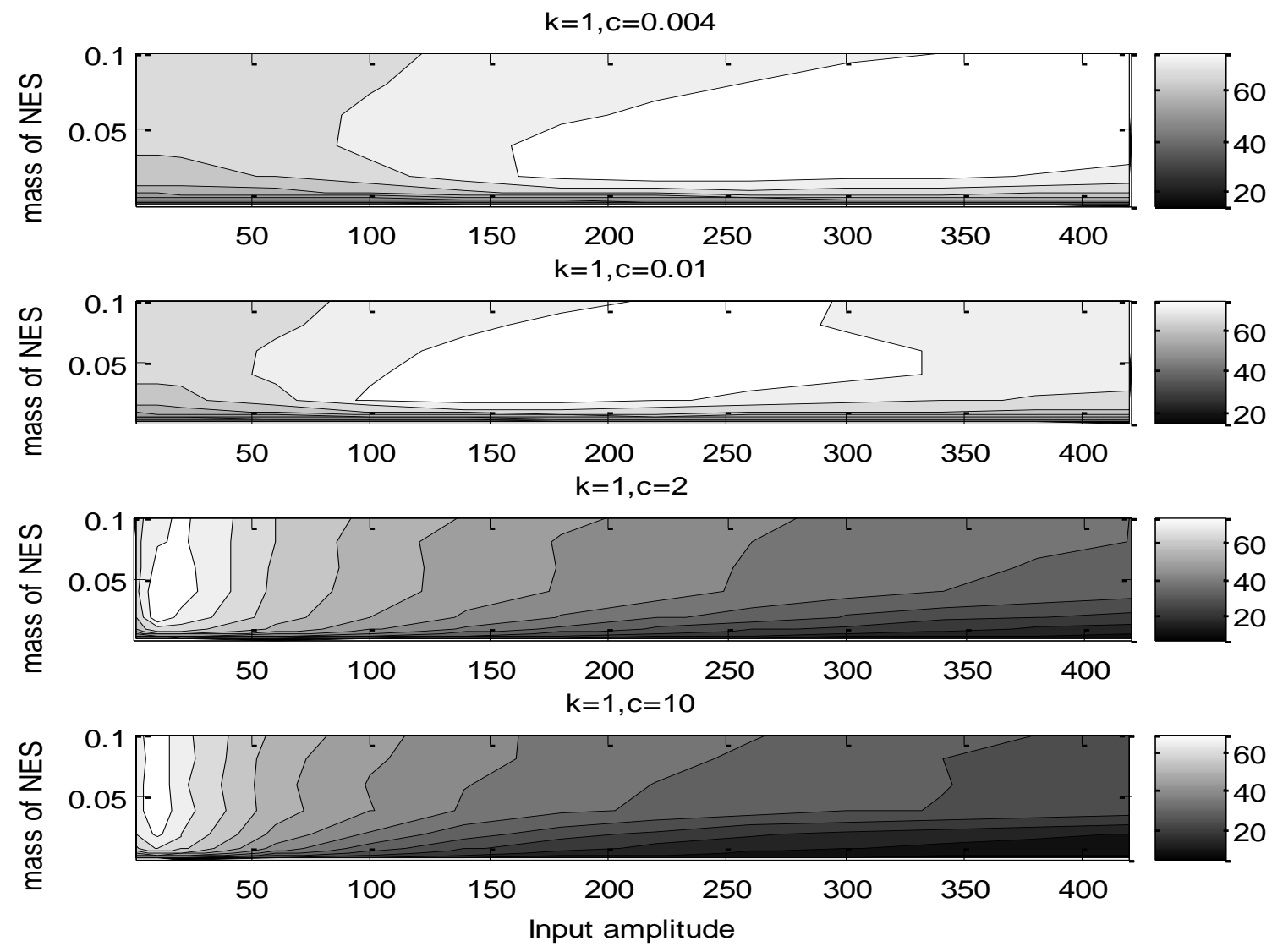

Figure 3 

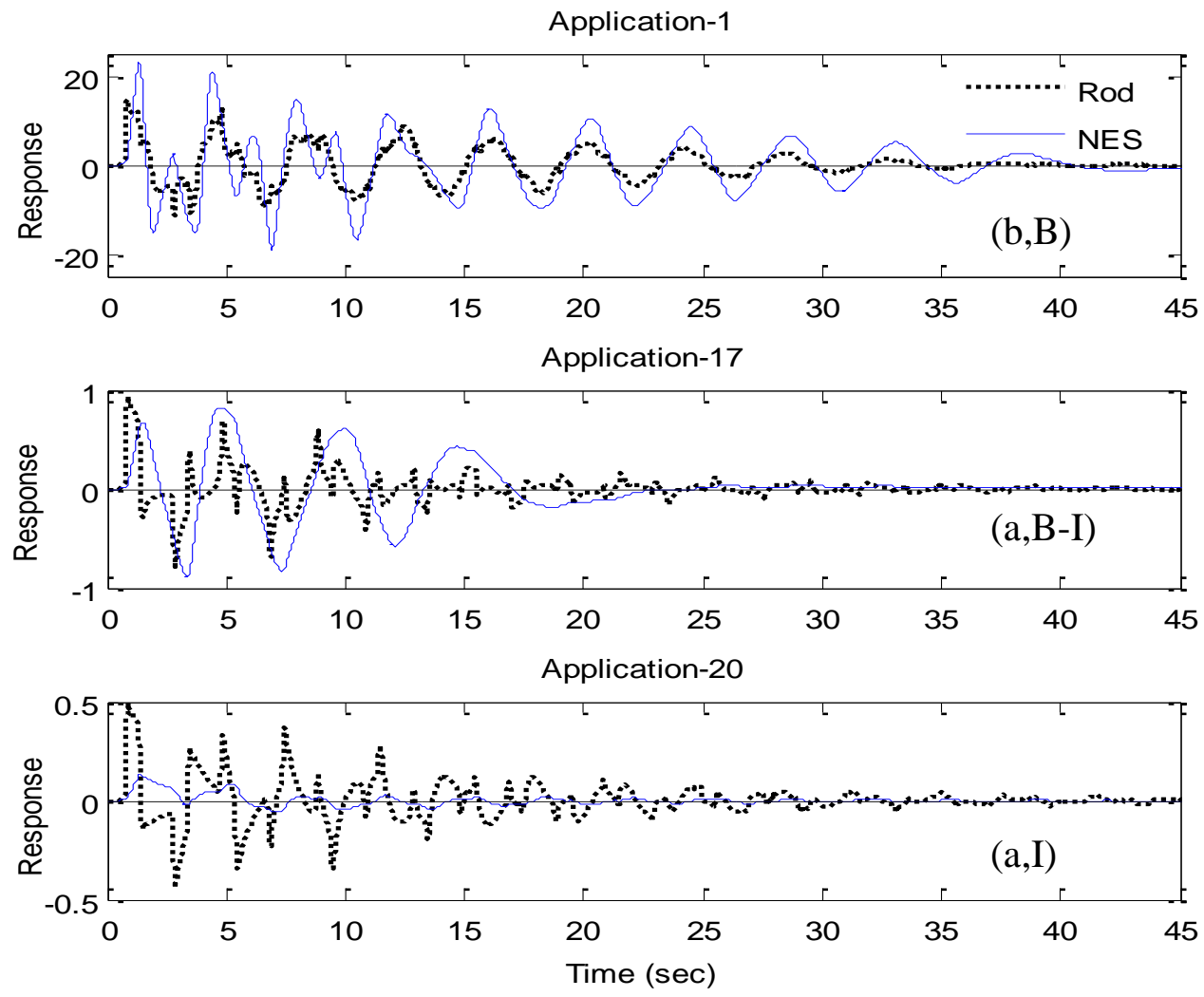

Figure 4 

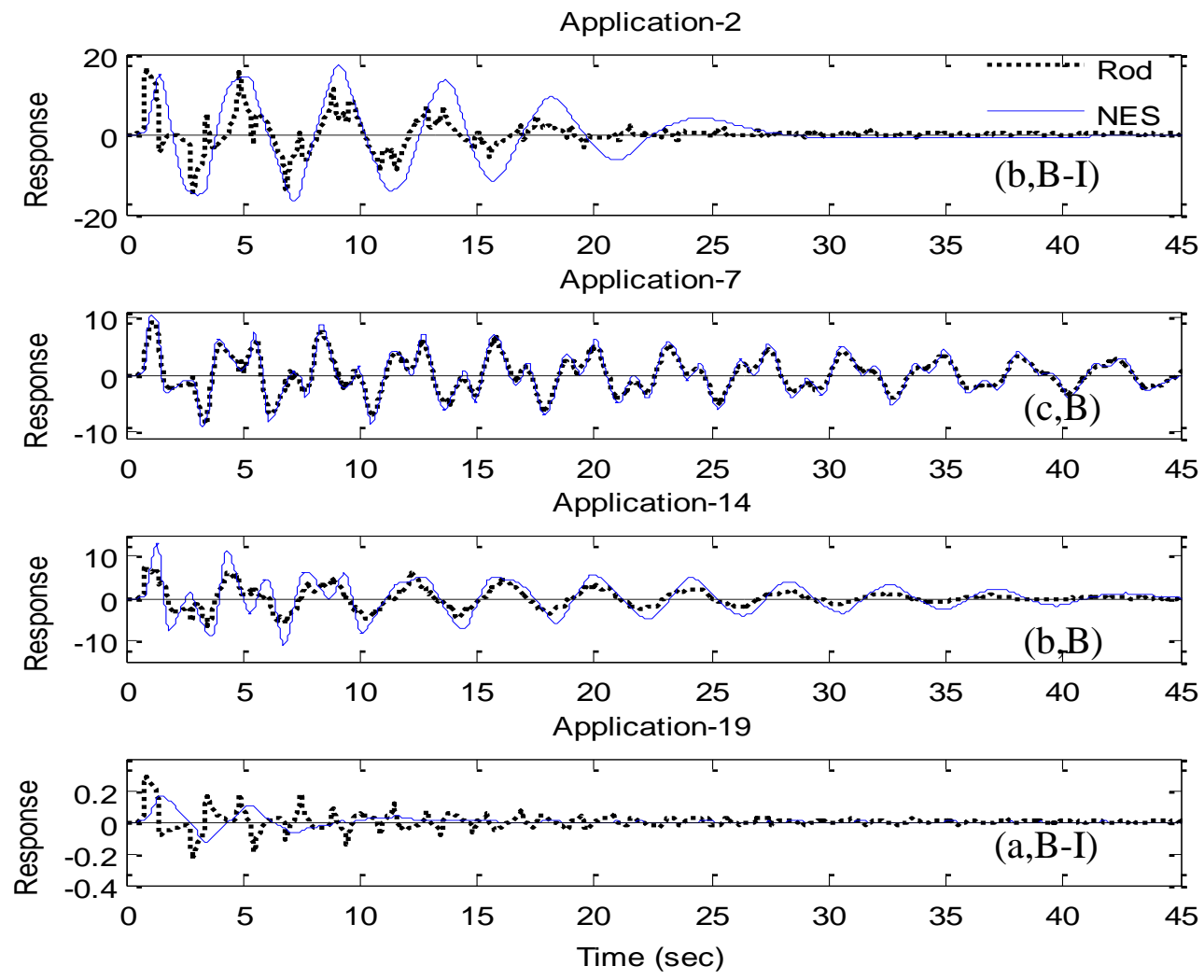

Figure 5 

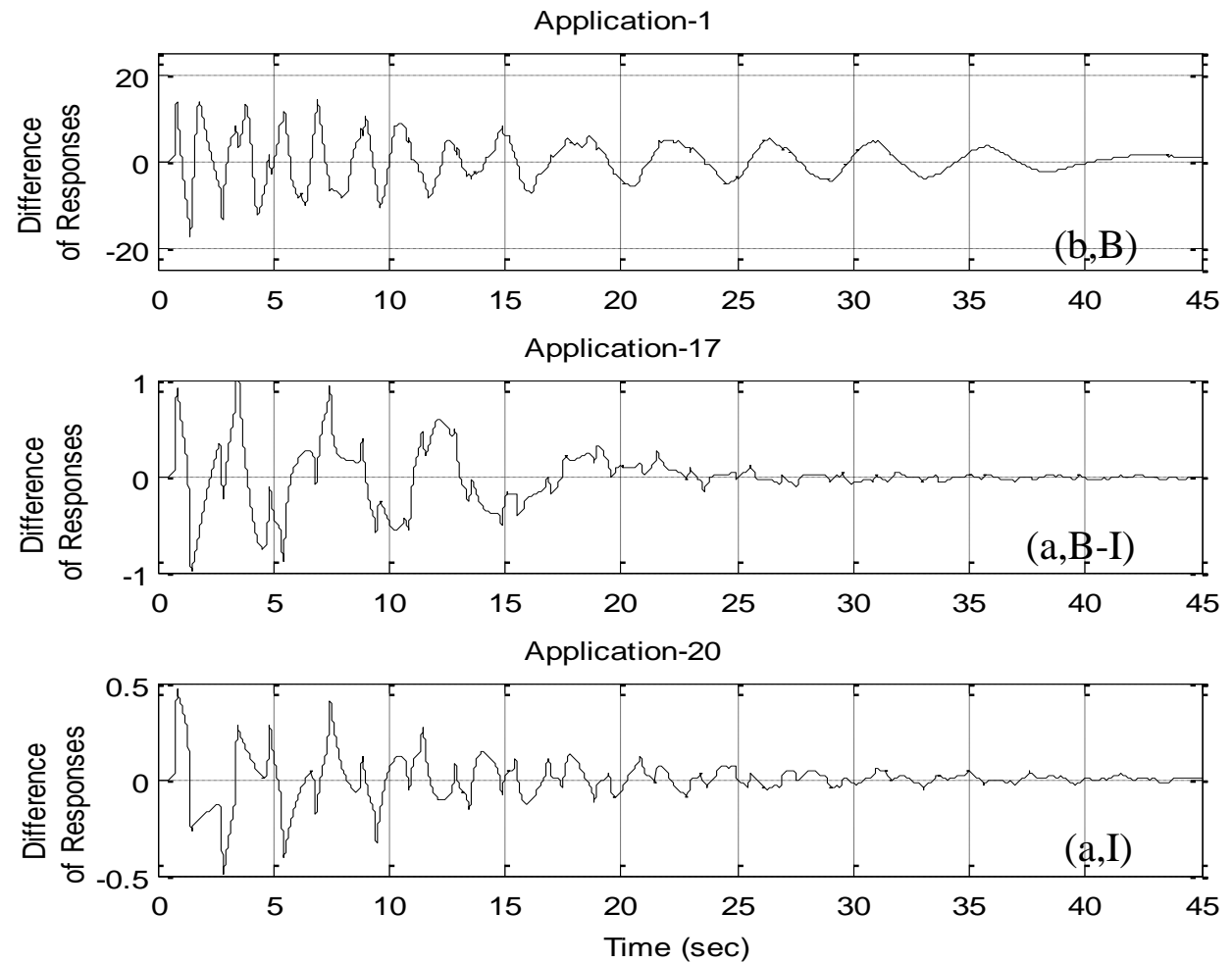

Figure 6
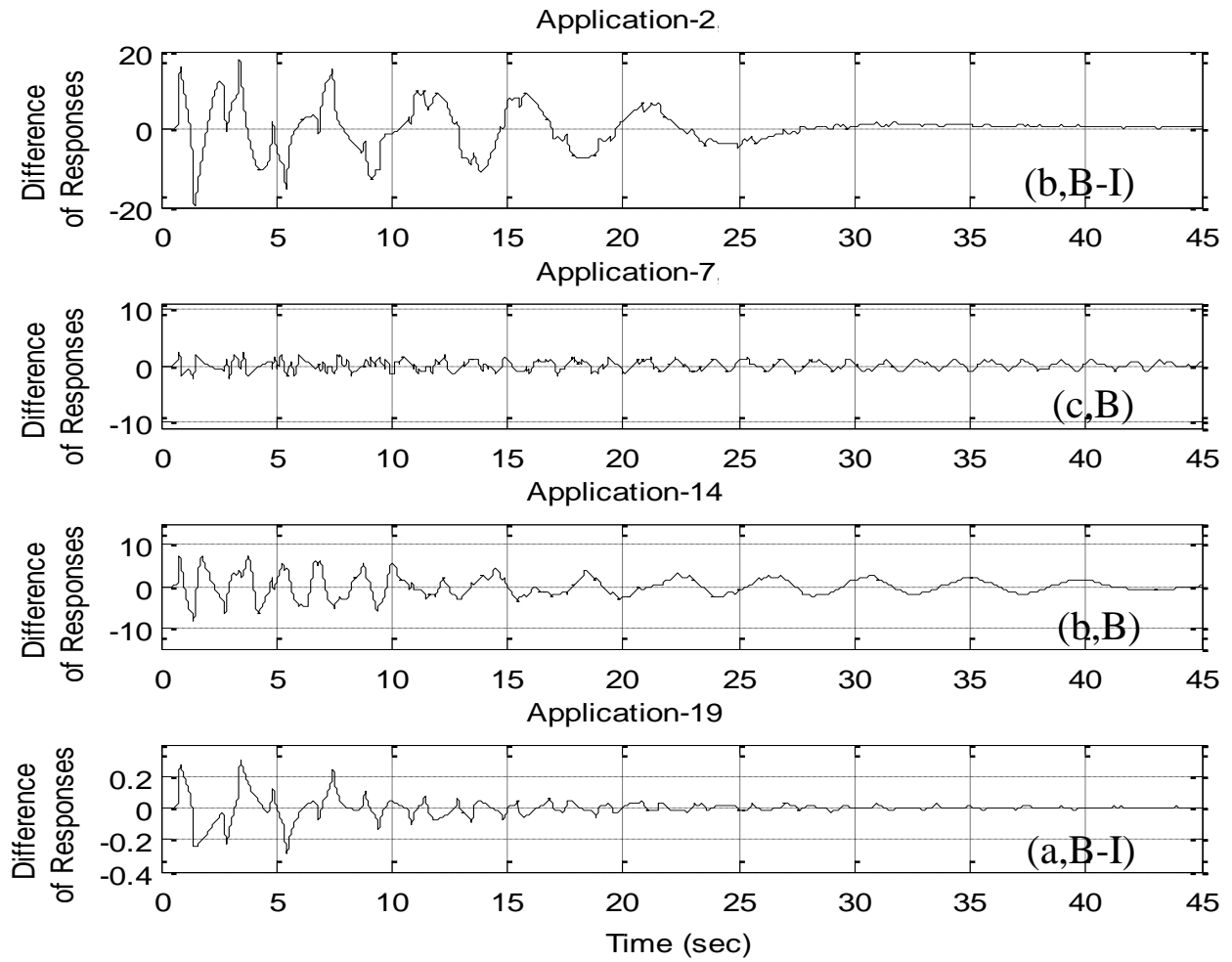

Figure 7 

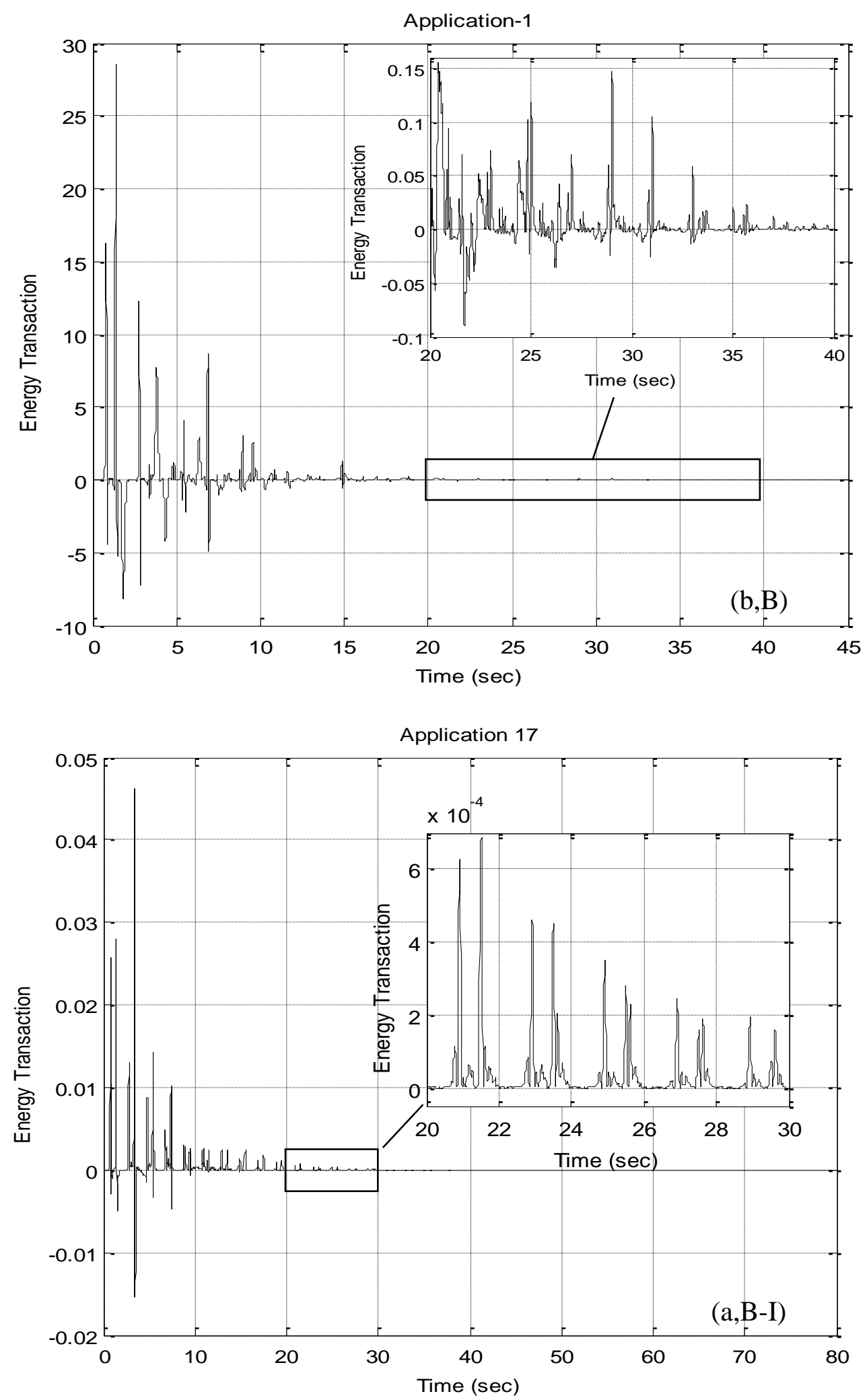

Figure 8 

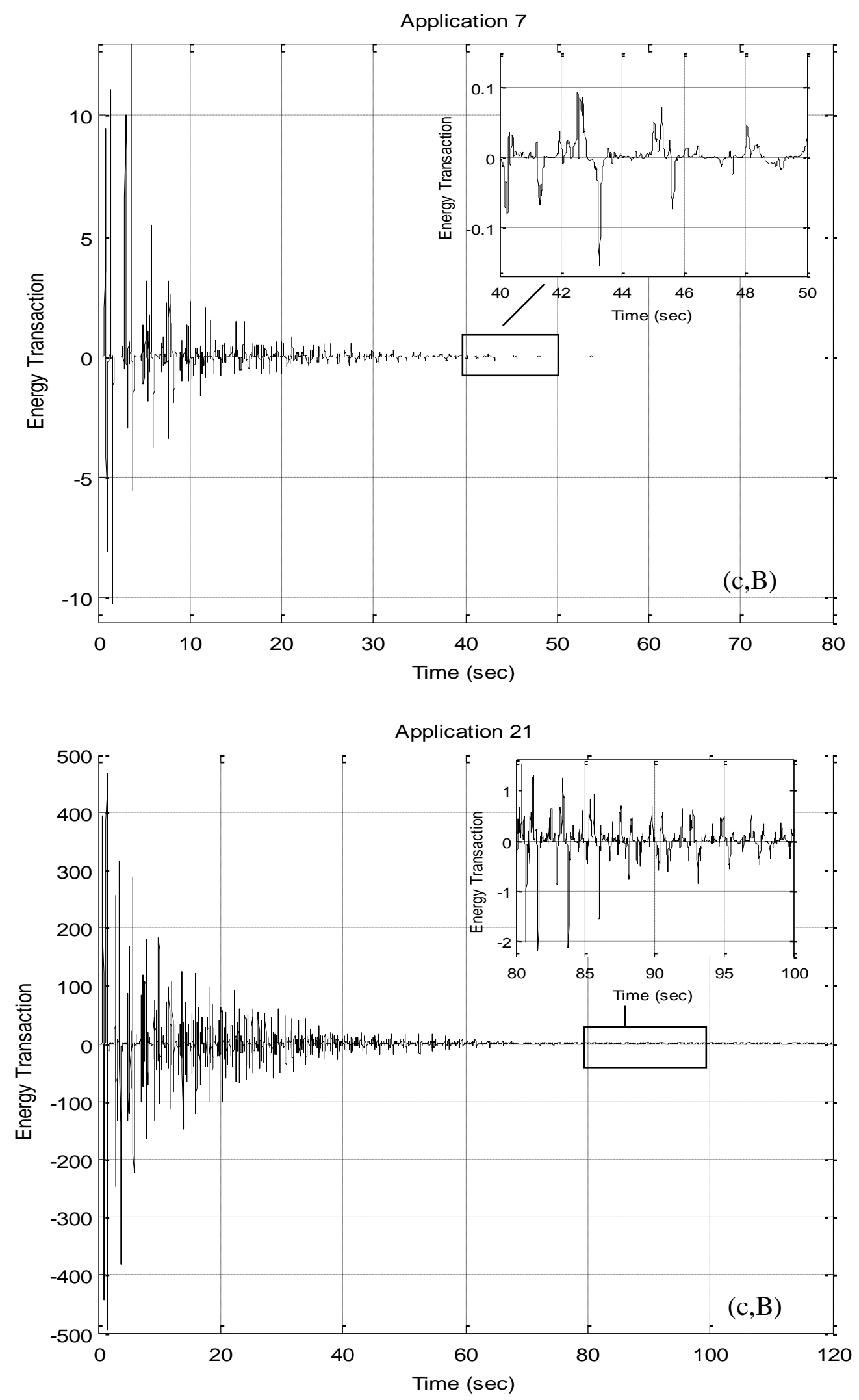

Figure 9 


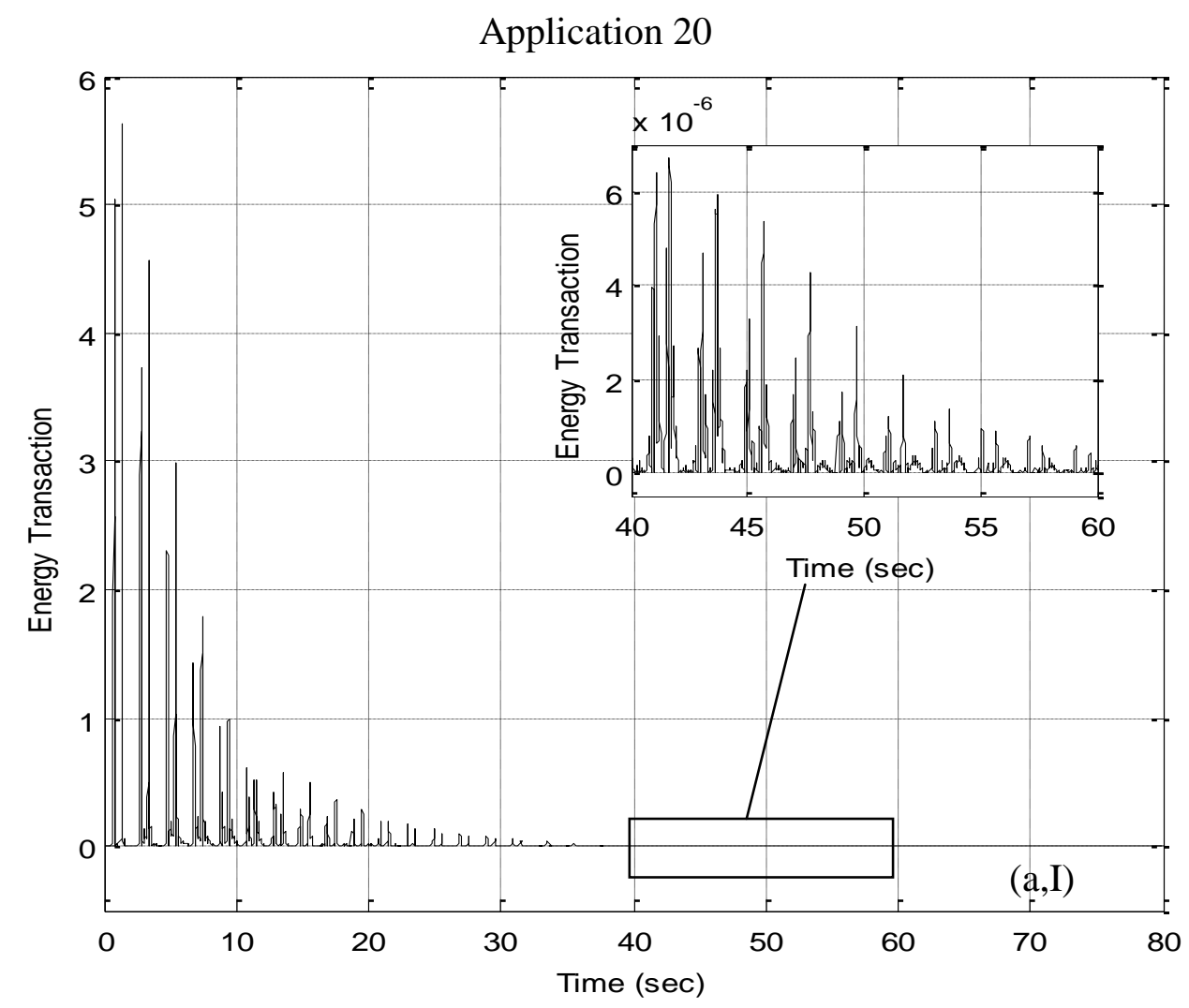

Figure 10 
(a)
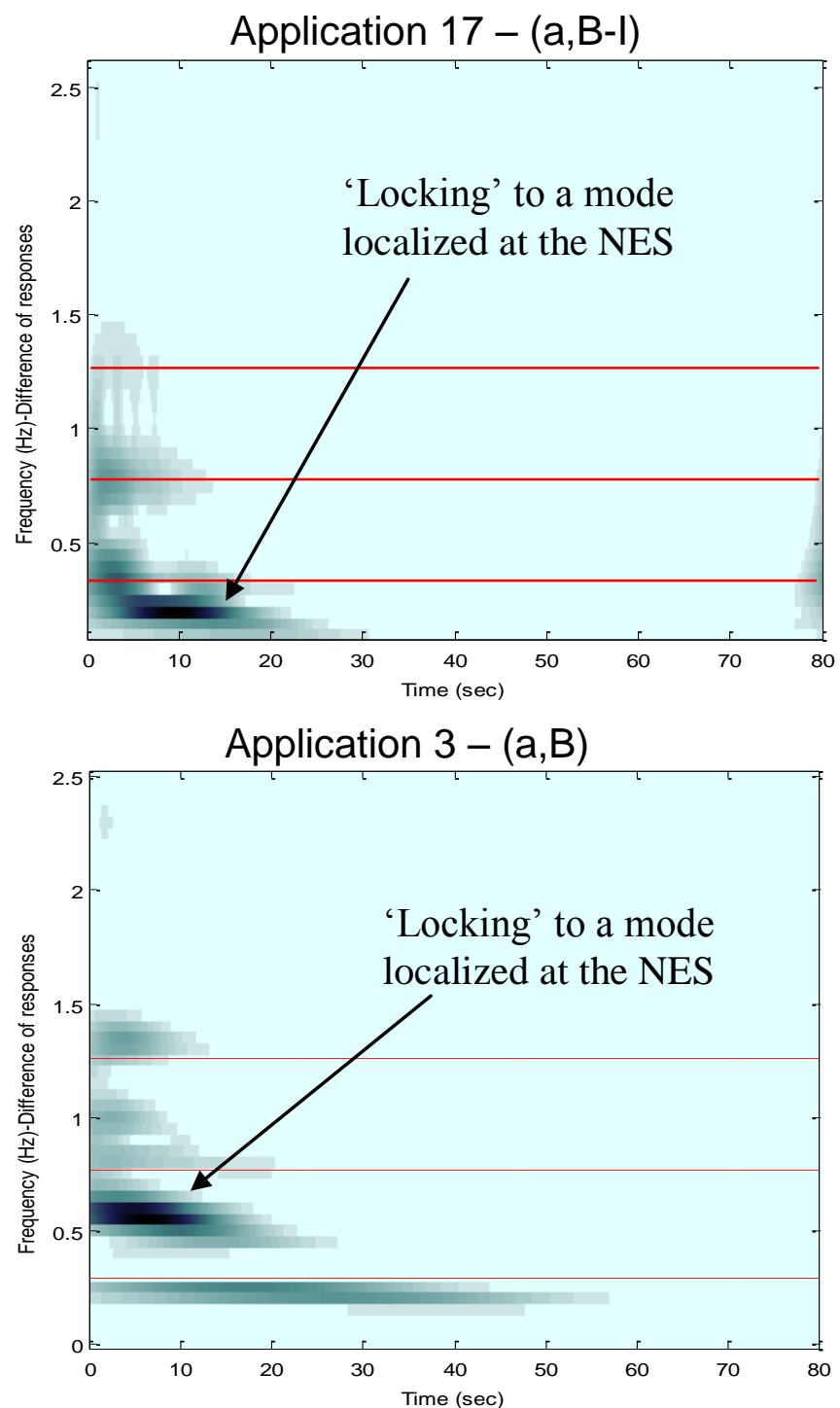

(b)

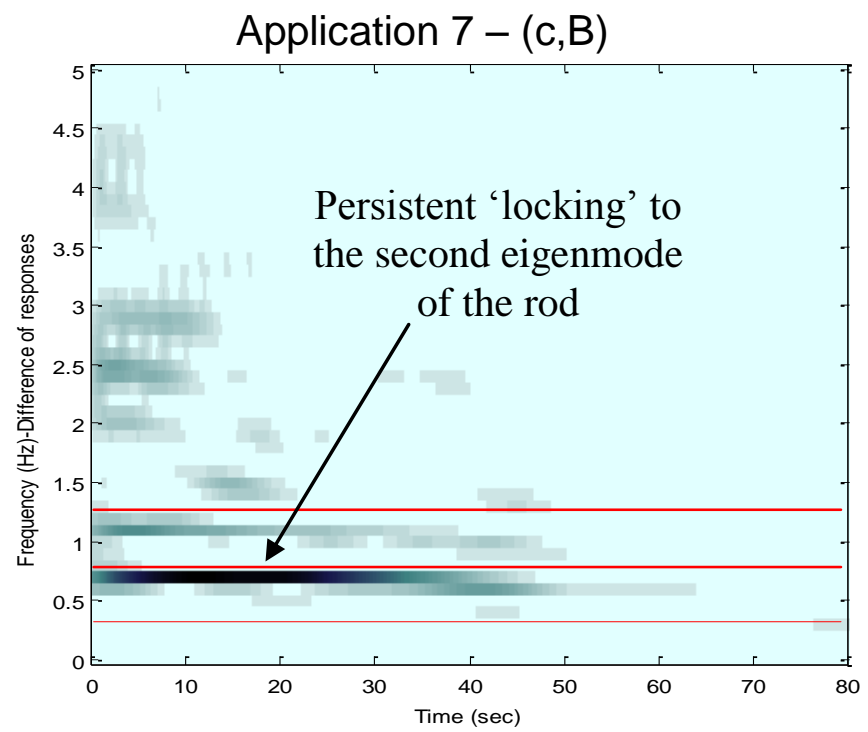

Figure $11(a, b, c)$ 


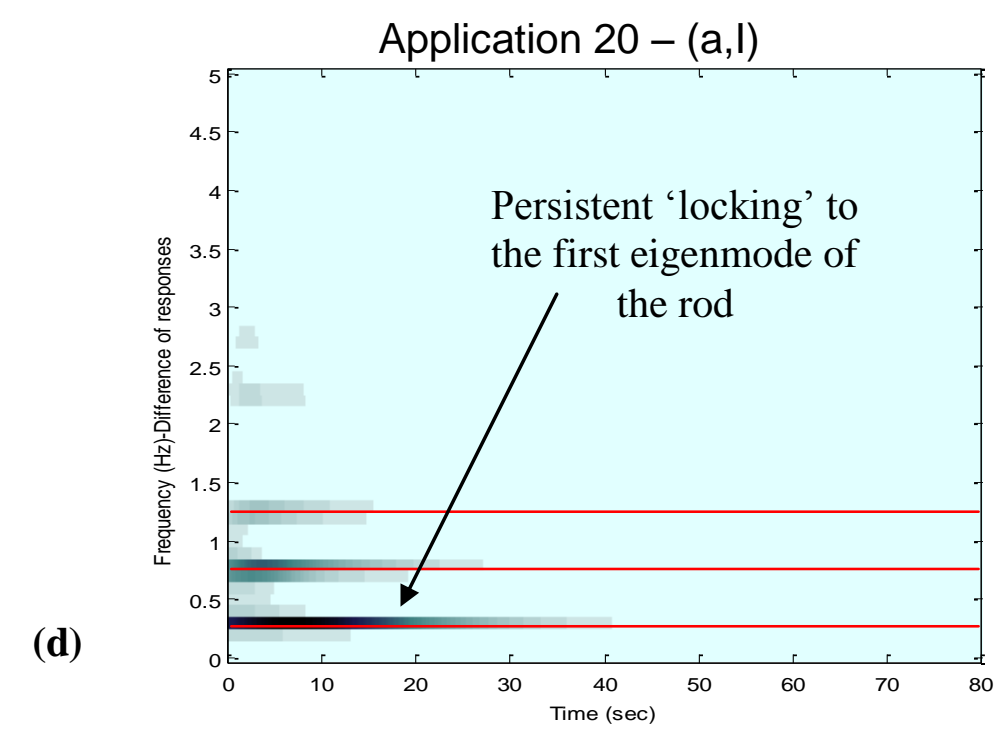

Figure 11(d) 

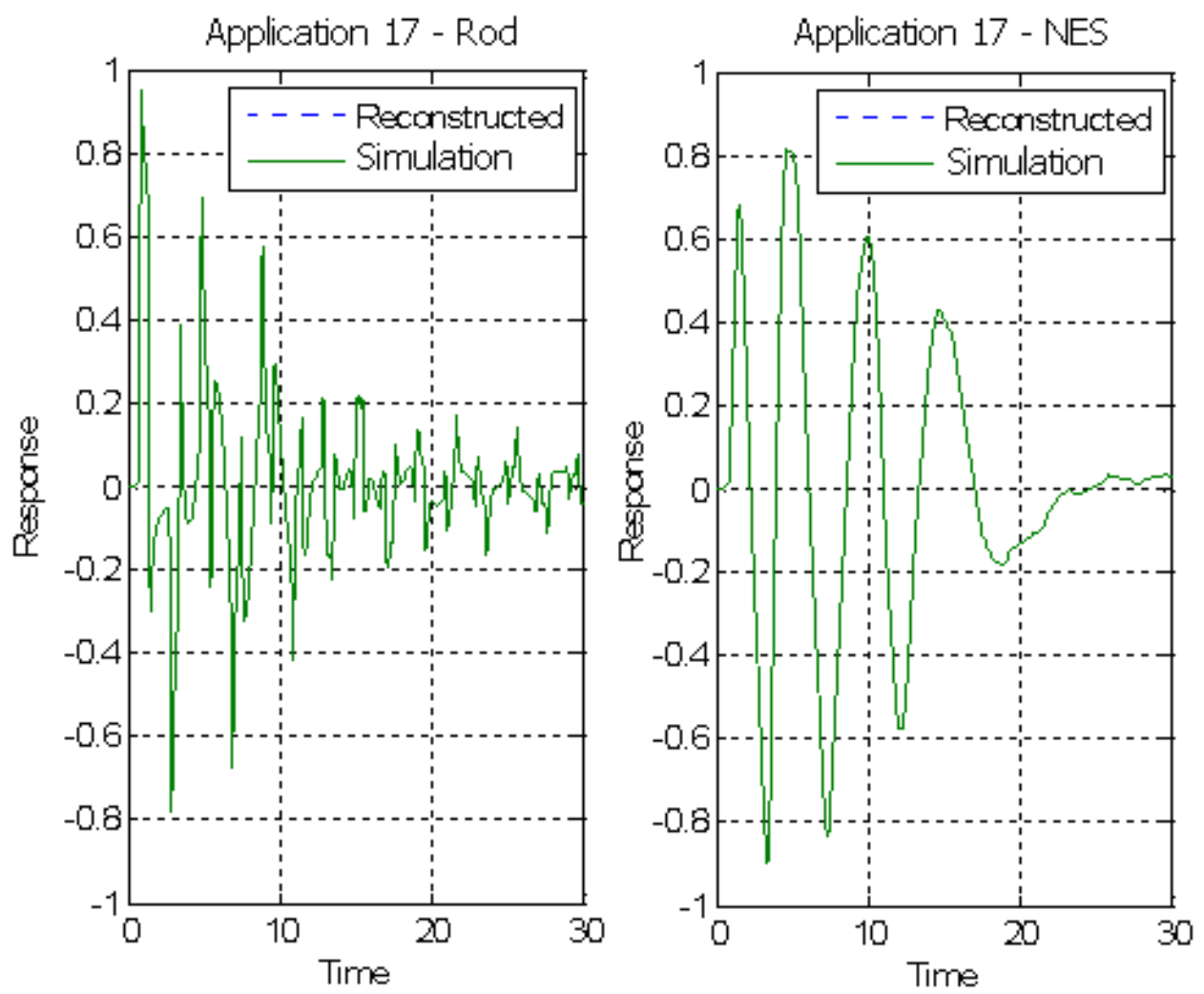

Figure 12(a) 

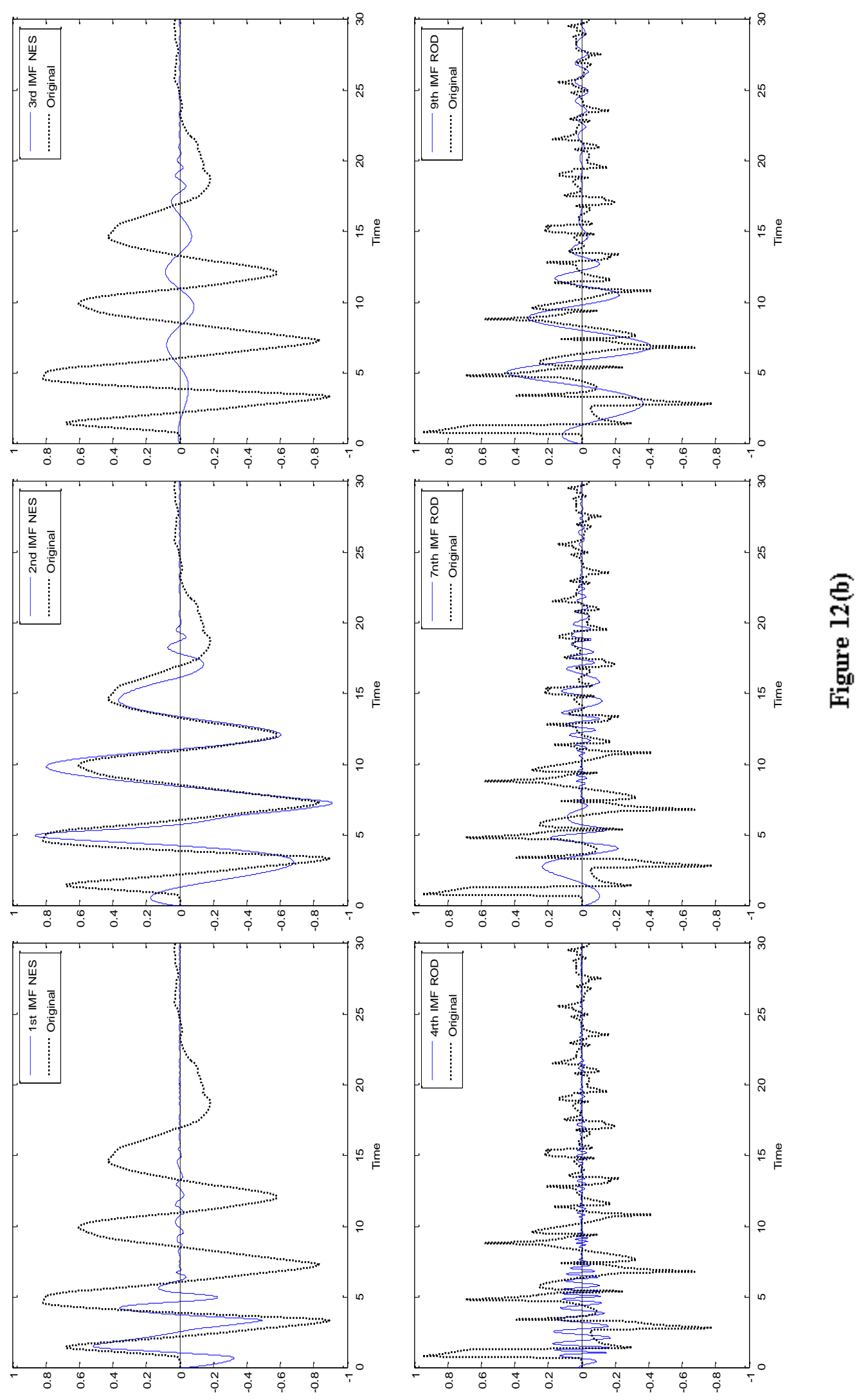

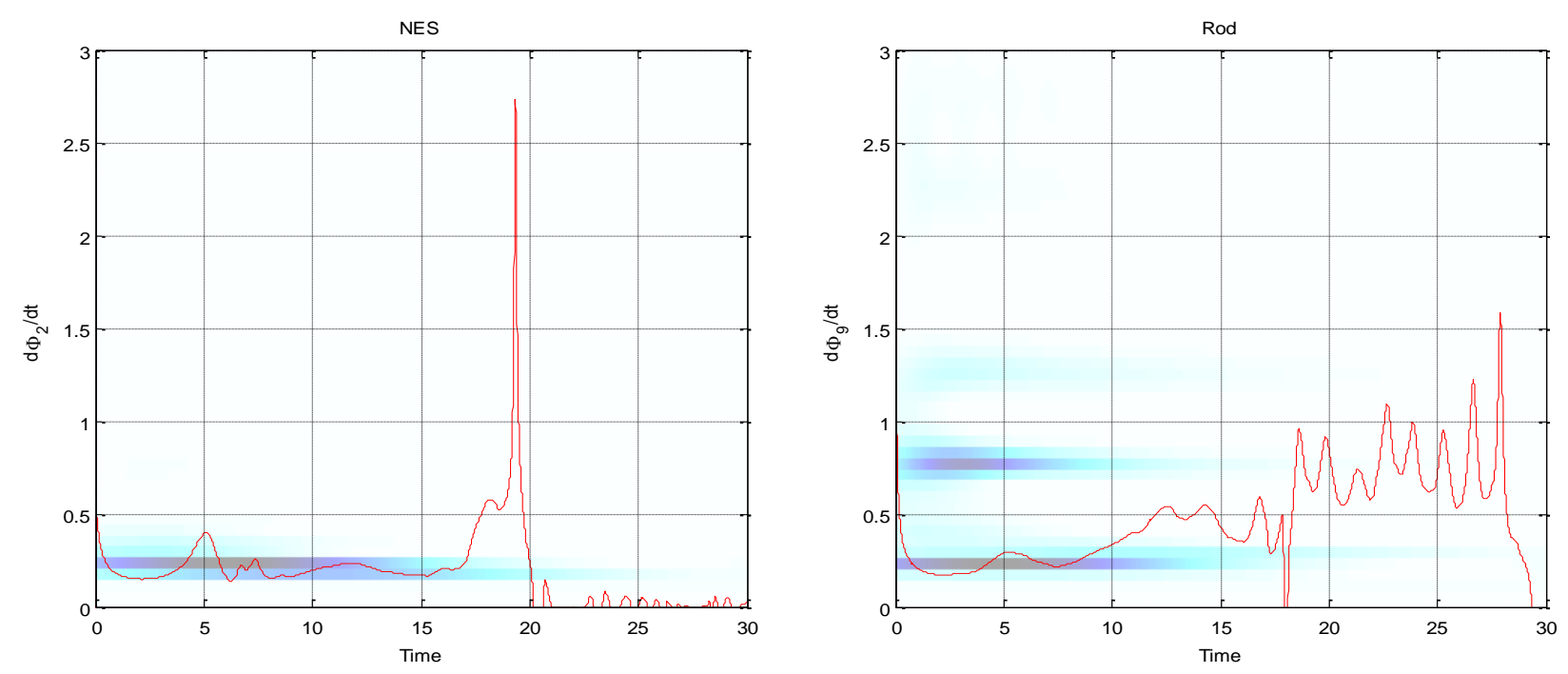

(a)

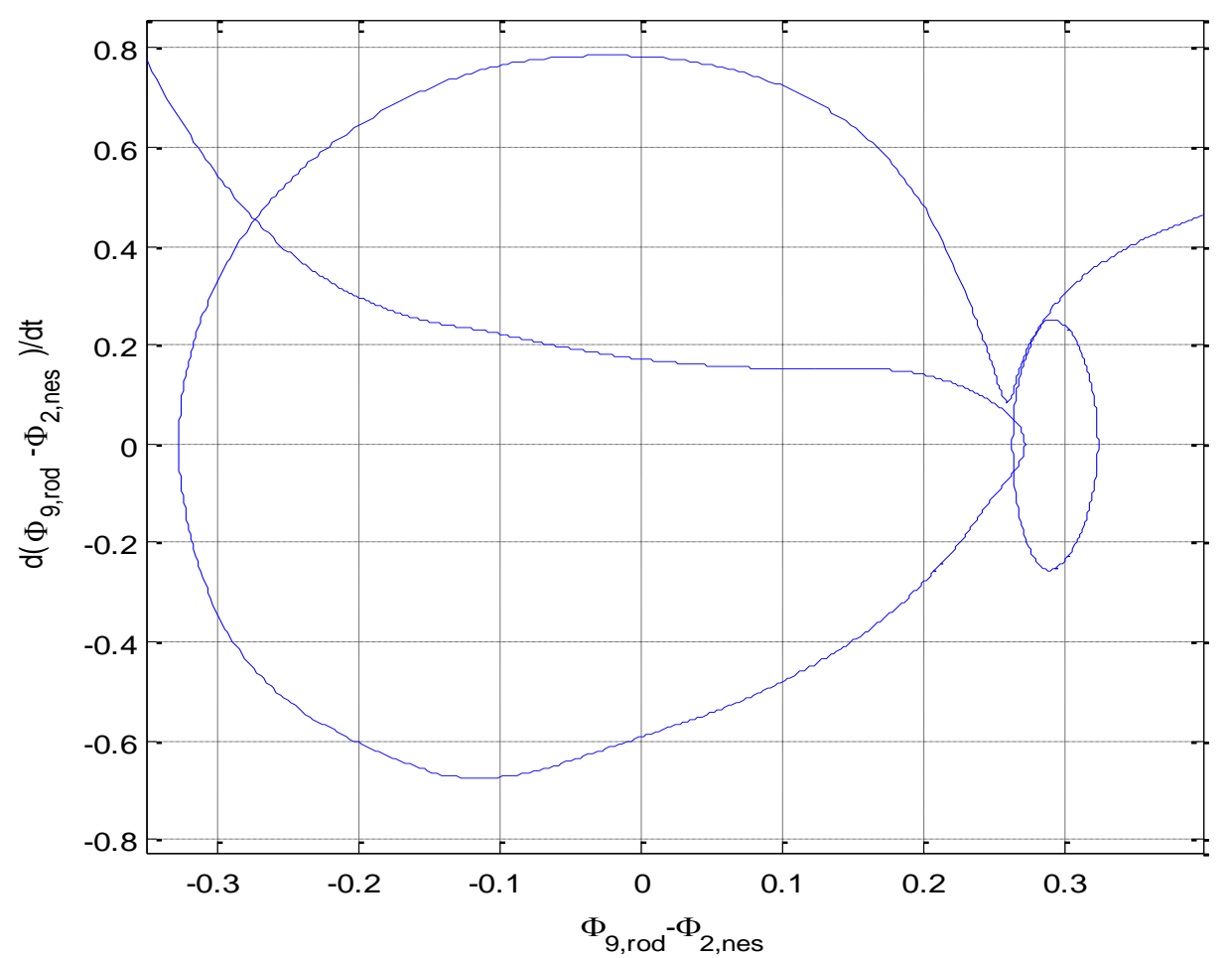

(b)

Figure 13 

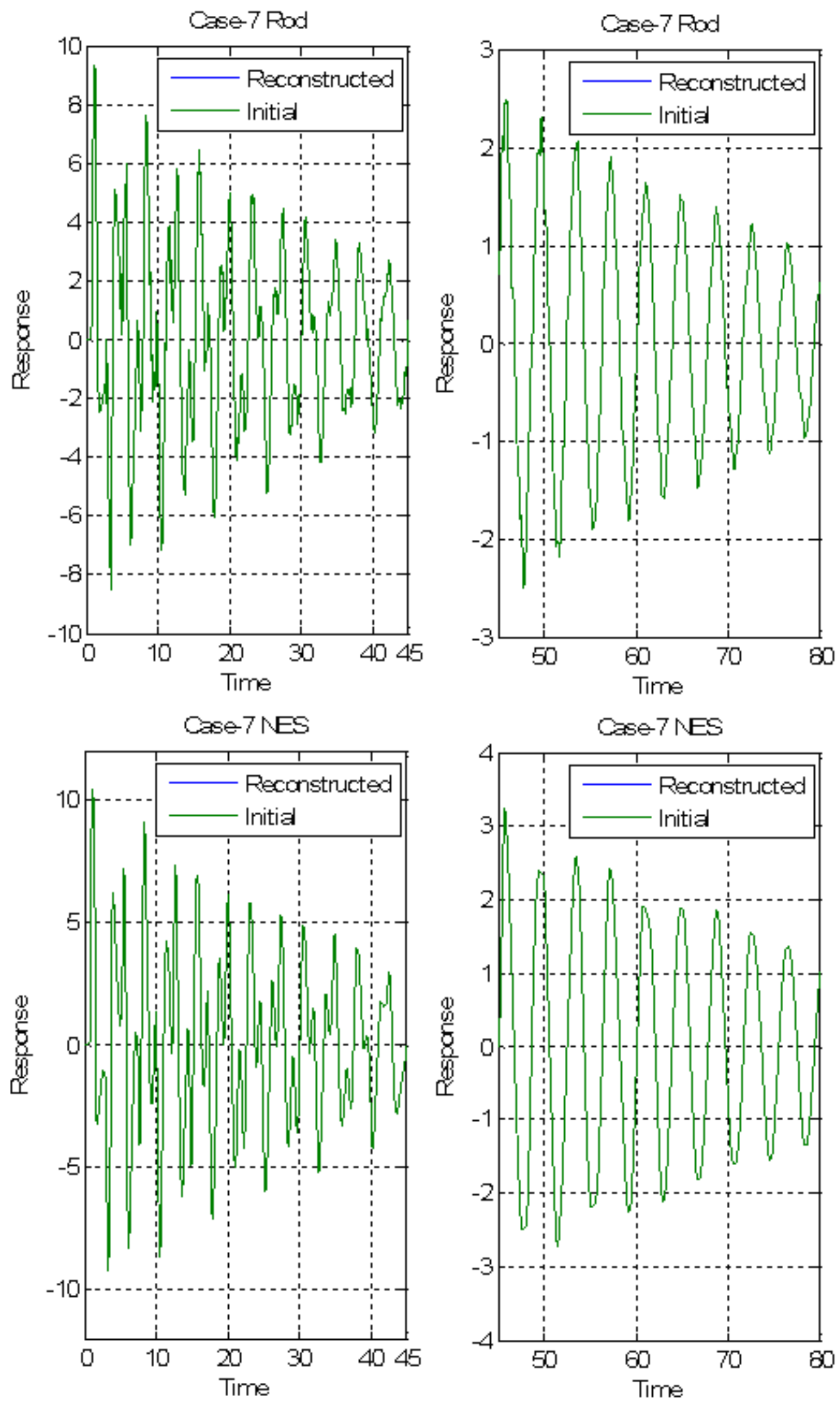

Figure 14(a) 

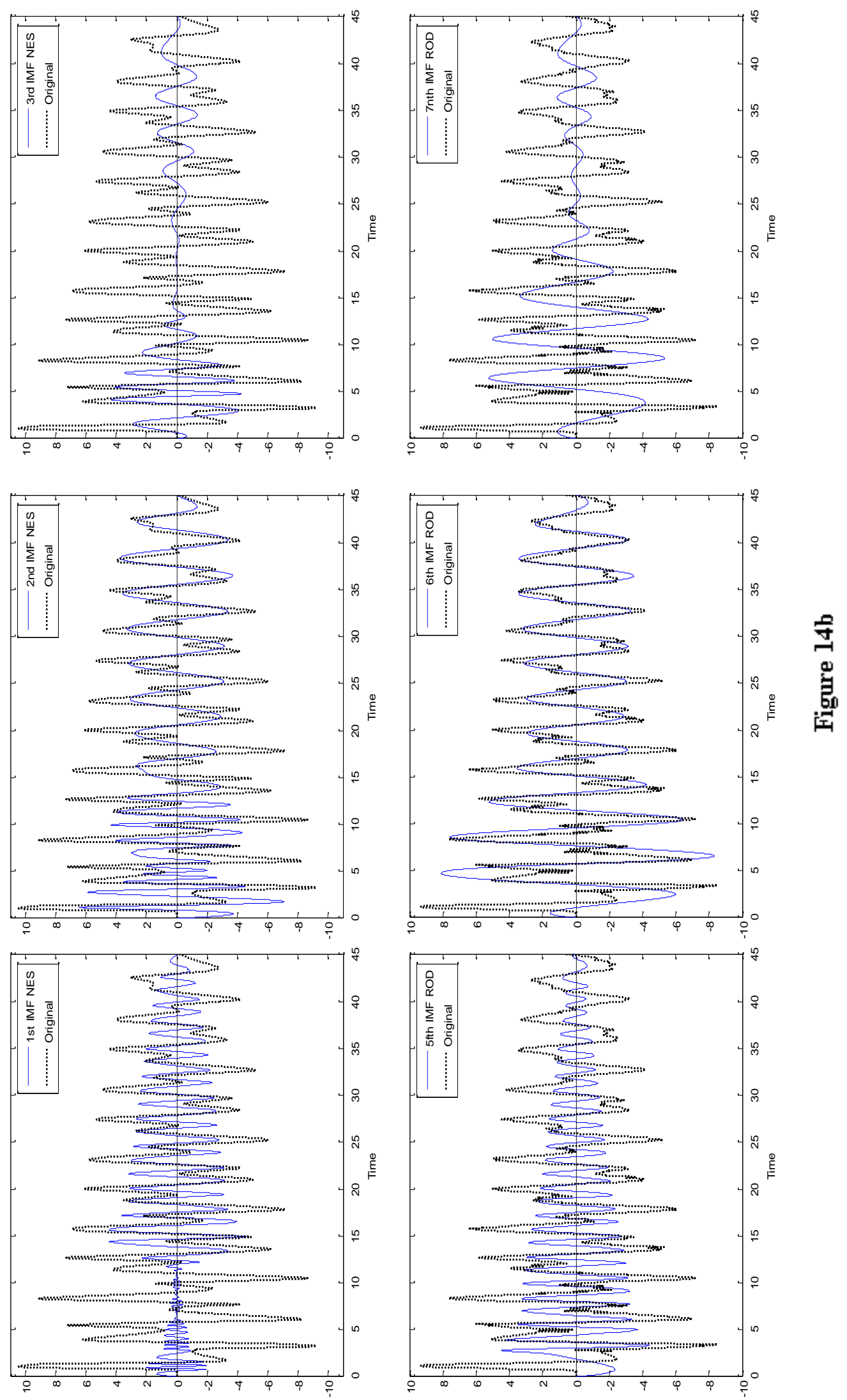

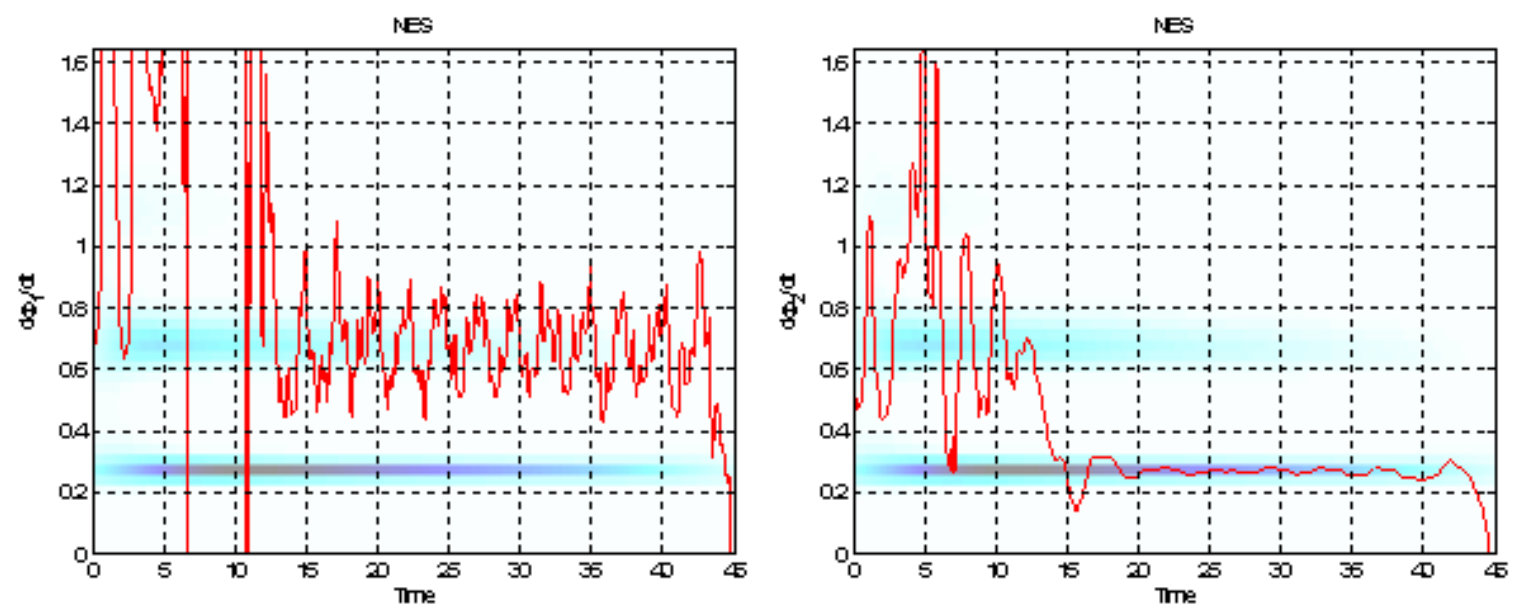

(a)
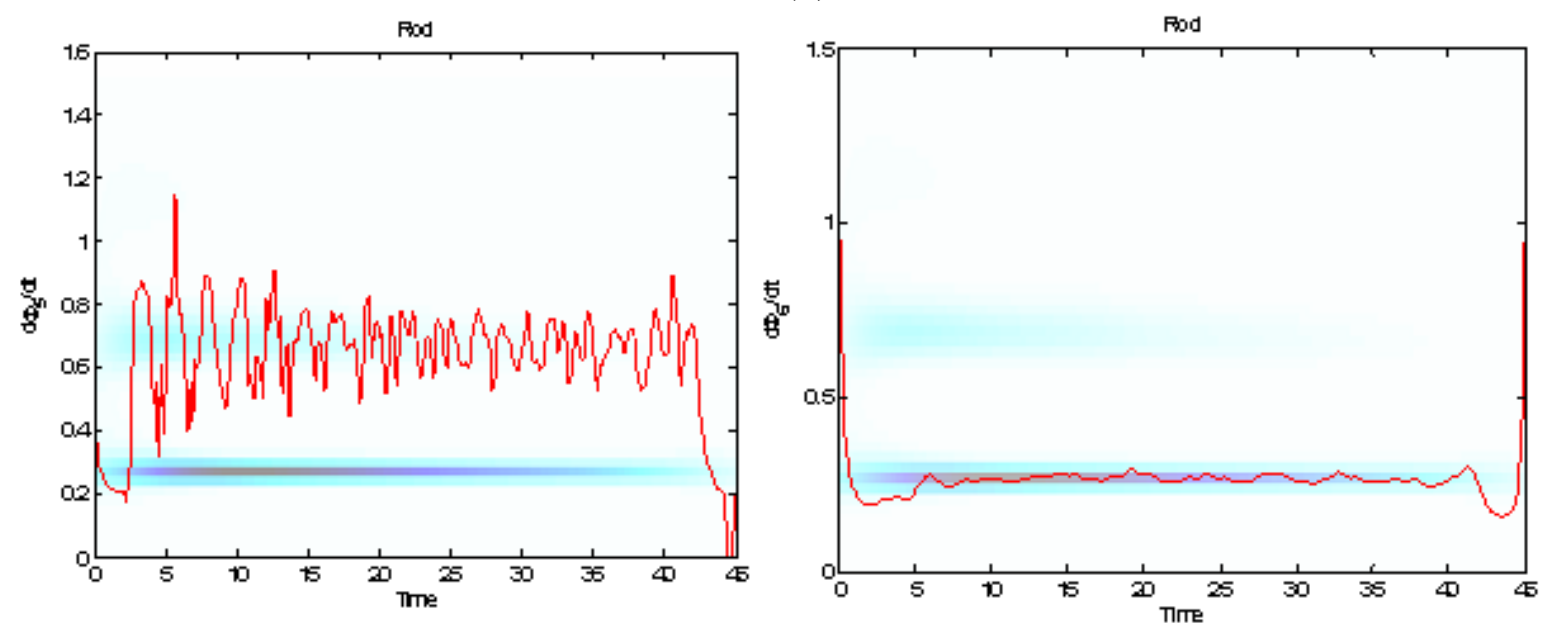

(b)
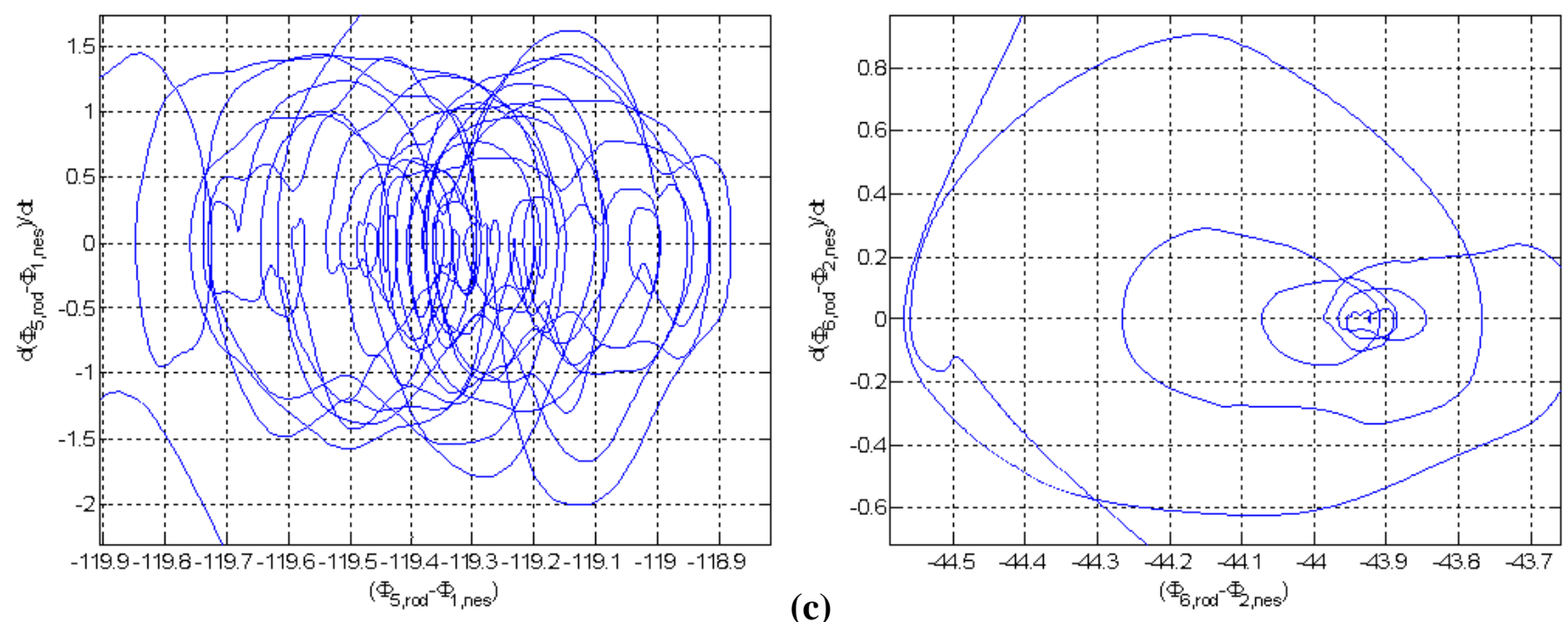

Figure 15 